\title{
Effect of Magnetic Twist on Nonlinear Transverse Kink Oscillations of Line-tied Magnetic Flux Tubes
}

\author{
J. Terradas ${ }^{1,2}$ (i), N. Magyar ${ }^{3}$, and T. Van Doorsselaere ${ }^{3}$ \\ ${ }^{1}$ Departament de Física, Universitat de les Illes Balears (UIB), E-07122, Spain; jaume.terradas@uib.es \\ ${ }^{2}$ Institute of Applied Computing \& Community Code (IAC $\left.{ }^{3}\right)$, UIB, Spain \\ ${ }^{3}$ Centre for Mathematical Plasma Astrophysics Department of Mathematics, Celestijnenlaan 200B, B-3001, KULeuven, Leuven, Belgium \\ Received 2017 May 24; revised 2017 November 15; accepted 2017 November 22; published 2018 January 19
}

\begin{abstract}
Magnetic twist is thought to play an important role in many structures of the solar atmosphere. One of the effects of twist is to modify the properties of the eigenmodes of magnetic tubes. In the linear regime standing kink solutions are characterized by a change in polarization of the transverse displacement along the twisted tube. In the nonlinear regime, magnetic twist affects the development of shear instabilities that appear at the tube boundary when it is oscillating laterally. These Kelvin-Helmholtz instabilities (KHI) are produced either by the jump in the azimuthal component of the velocity at the edge of the sharp boundary between the internal and external part of the tube or by the continuous small length scales produced by phase mixing when there is a smooth inhomogeneous layer. In this work the effect of twist is consistently investigated by solving the time-dependent problem including the process of energy transfer to the inhomogeneous layer. It is found that twist always delays the appearance of the shear instability, but for tubes with thin inhomogeneous layers the effect is relatively small for moderate values of twist. On the contrary, for tubes with thick layers, the effect of twist is much stronger. This can have some important implications regarding observations of transverse kink modes and the KHI itself.
\end{abstract}

Key words: magnetohydrodynamics (MHD) - Sun: magnetic fields - waves

\section{Introduction}

Standing transverse oscillations of cylindrical magnetic tubes are prone to develop, in the nonlinear regime, shear instabilities at the tube boundary (e.g., Terradas et al. 2008; Antolin et al. 2014, 2015; Magyar et al. 2015; Magyar \& Van Doorsselaere 2016; Antolin et al. 2017). The instability can take place in magnetic tubes with either a sharp or a smooth density transition between the internal part of the tube and the lighter external medium.

For a sharp transition and in the linear regime, eigenmode calculations show that the azimuthal component of the velocity is discontinuous at the tube radius (see, e.g., Goossens et al. 2012). This is the origin of the Kelvin-Helmholtz instability (KHI) found in the nonlinear regime. The velocity shear profile is rather different from the interface equilibrium model used to investigate this instability based on stationary and invariant flows (see, e.g., Chandrasekhar 1961). For a cylindrical magnetic tube oscillating with the standing transverse $m=1$ mode, the shear flow due to the oscillation is sinusoidal in the azimuthal and longitudinal direction (along the axis of the tube). Since the tube is oscillating, the flow is not stationary in time and has the periodicity of the kink oscillation. This complicates significantly an analytical study of the problem even in the linear regime, and numerical simulations are in general required to address this problem.

For a smooth transition at the tube boundary the situation is even more complex. Transverse kink oscillations are attenuated owing to the energy transference to the inhomogeneous layer by the mechanism of resonant damping (e.g., Hollweg \& Yang 1988; Ruderman \& Roberts 2002; Goossens et al. 2002). Sakurai et al. (1991) and Goossens et al. (1992) showed that the perpendicular component of the velocity at the resonant position $\left(r=r_{\mathrm{A}}\right)$ has a $1 / s$ singularity (where $s=r-r_{\mathrm{A}}$ ) and a $\delta(s)$ contribution. Later, Goossens et al. (1995) showed how dissipation smooths the velocity profile (see their Figure 1). The bulk transverse motion of the tube is eventually transformed into highly localized shear motions at the edge of the tube where phase mixing takes place. In comparison with the sharp transition configuration, there is now an additional complication, the change in time of the shear profile in the radial direction. Decreasing spatial scales are continuously generated at the inhomogeneous layer by the phase-mixing process.

Heyvaerts \& Priest (1983) and Browning \& Priest (1984) were able to study the development of the KHI in a very simple configuration and for uncoupled Aflvén waves $(m=0)$. In their model, the process of resonant damping is not considered and the amount of energy in the layer does not grow with time. However, their results can be used as a crude guide to better understand the onset of the KHI. These authors performed a temporally local stability analysis and found a critical time after which the instability grows significantly within one period of oscillation. In essence the authors solve the standard Rayleigh stability equation for a shear flow in two different regimes. The first regime is characterized by length scales produced by phase mixing that are much shorter than the typical inhomogeneity length scale. In this strong phase-mixing situation the shear profile is approximated by a rapidly oscillating sinusoidal function (Browning \& Priest 1984). The instability could, however, develop before the situation of strong phase mixing is reached. In this regime the instability at first grows slowly, but after a critical time the instability develops within one wave period. The critical time depends on the ratio of the inhomogeneity scale length to the wavelength parallel to the equilibrium magnetic field.

The main aim of the present work is to study the stabilizing effect of an azimuthal component of the magnetic field on the development of the KHI. In this regard, Soler et al. (2010) performed an analytical study considering the linear stage of 
the KHI in a cylindrical magnetic tube in the presence of an invariant azimuthal flow, and one of the main conclusions was that, in principle, a small amount of twist should be able to suppress the instability. Zaqarashvili et al. (2015) have recently studied the stability of twisted and rotating jets caused by the velocity jump near the jet surface and have derived the dispersion relations for incompressible plasma. In these two studies the azimuthal flow is assumed to be invariant in the azimuthal direction and stationary in time in order to find analytic dispersion relations. However, in reality the transverse oscillations of a tube are much more involved.

Here, the full time-dependent problem is solved, involving the generation of small length scales in the inhomogeneous layer plus the continuous energy transference to this layer. This process is investigated by means of numerical simulations providing unique details about the onset of the KHI for different magnetic tube configurations. The method adopted here does not suffer from the limitations of the models of Heyvaerts \& Priest (1983) and Browning \& Priest (1984) and is not restricted to the stationary state of the system as in Soler et al. (2010) and Zaqarashvili et al. (2015). This allows us to perform, for the first time, a quantitative analysis of the effect of magnetic twist on the development of the KHI in the context of line-tied oscillating magnetic flux tubes, representing, for example, coronal loops, which undergo resonant damping while they are oscillating.

\section{Cylindrical Twisted Flux Tube Model}

We consider a straight twisted magnetic tube of radius $R$ and length $L$ homogeneous in the axial direction. The equilibrium magnetic field depends on $r$ only and has two components, axial, $B_{z}$, and azimuthal, $B_{\phi}$. The force-free condition in the absence of pressure gradients (we assume a constant gas pressure) reduces to

$$
\frac{d B^{2}}{d r}=-\frac{2 B_{\phi}^{2}}{r},
$$

where $B^{2}=B_{z}^{2}+B_{\phi}^{2}$. We assume that $B_{z e}$ is constant, $B_{\phi i}$ is proportional to $r, B_{\phi e}$ is inversely proportional to $r$, and the magnetic field is continuous at $r=R$ (the subscripts $e$ and $i$ refer to the external and internal values, respectively). This model has been recently used by Ruderman \& Terradas (2015) and Giagkiozis et al. (2016). Then, using Equation (1), we obtain that the equilibrium magnetic field is given by

$$
\begin{gathered}
B_{z}^{2}=\left\{\begin{array}{cc}
B_{0}^{2}+2 A^{2}\left(1-r^{2} / R^{2}\right), & r<R, \\
B_{0}^{2}, & r>R,
\end{array}\right. \\
B_{\phi}= \begin{cases}A r / R, & r<R . \\
A R / r, & r>R .\end{cases}
\end{gathered}
$$

where $A$ and $B_{0}$ are positive constants. In this model the ratio of $B_{\phi} / B_{z}$ at $r=R$ is $A / B_{0}$. In the present work four different values of twist are considered, $B_{\phi} / B_{z}=[0,0.1,0.2,0.3,0.4]$ (at $r=R$ ), and the corresponding twist angles are $\theta=[0,5.7$, $11.3,16.7,21.8]$ (in degrees). The number of turns over the cylinder length $L$ is defined as

$$
N_{t w}=\frac{1}{2 \pi} \frac{L}{r} \frac{B_{\phi}}{B_{z}} .
$$

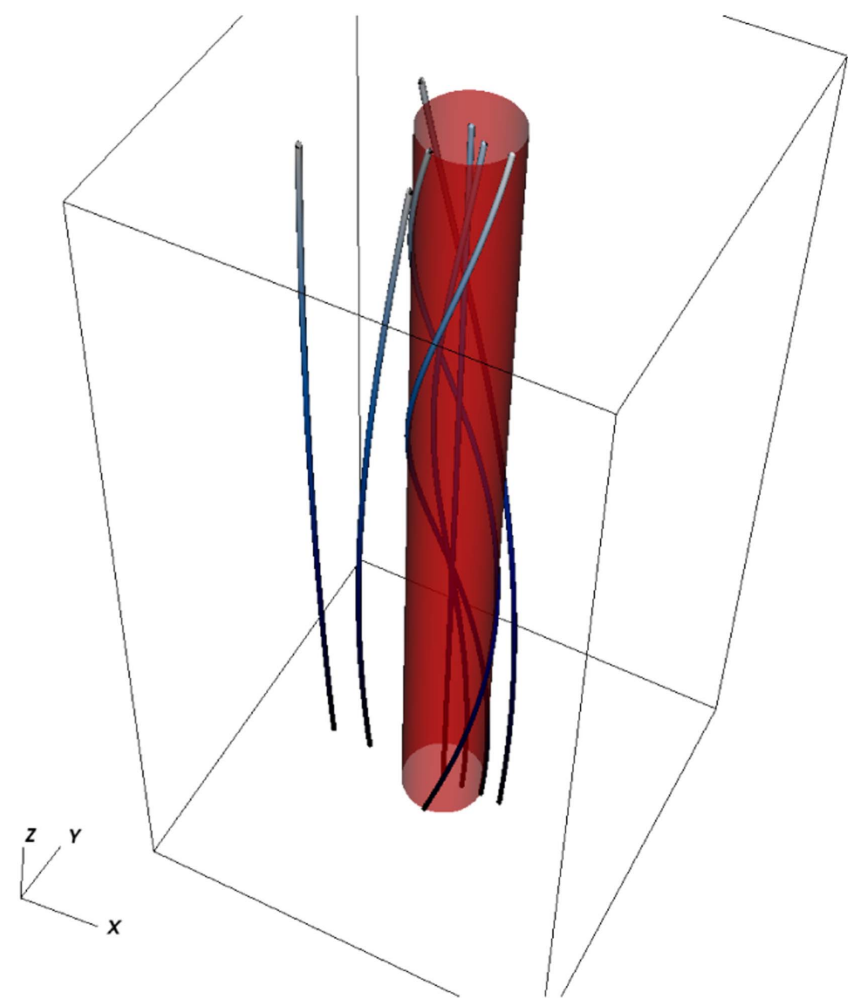

Figure 1. Sketch of the equilibrium model. Some specific magnetic field lines for the case $B_{\phi} / B_{z}=0.3$ are plotted in blue. The maximum number of turns over the cylinder is 0.95 . The density isocontour is plotted in red and corresponds to $r=R$.

At $r=R$, where twist has a maximum, we have that $N_{t w}=$ $[0,0.32,0.64,0.95,1.27]$ (assuming that $L=20 R$ ). An example of the magnetic field configuration is shown in Figure 1. We have intentionally avoided a higher number of turns because the magnetic tube might become kink unstable. In fact, according to the results of Hood \& Priest (1979), for a force-free field of uniform twist the critical twist is $3.3 \pi$, which is 1.65 turns. Therefore, we are not too far from this critical value. The range of values for the twist considered in this work is in agreement with the twist angles that are inferred from the estimations performed by Aschwanden (2013) and Aschwanden et al. (2014, 2016). These estimations are based on nonlinear force-free magnetic fields calculated using line-ofsight magnetograms and the geometry of observed coronal loops in 3D. The twist angle can be calculated from the expression $\theta=\operatorname{atan}\left(\sqrt{q_{\text {free }}}\right)$, where $q_{\text {free }}$ is the free magnetic energy ratio, which is typically in the range $0<q_{\text {free }}<0.2$ (see, e.g., Table 4 of Aschwanden et al. 2014), meaning that the twist angle is always below $24^{\circ}$.

The equilibrium plasma density, $\rho$, is assumed to be constant inside $\left(\rho_{i}\right)$ and outside the tube $\left(\rho_{e}\right)$, but with a smooth density transition of thickness $l$ in the radial direction. We use a sinusoidal profile to connect the internal and external regions of the tube (see, e.g., Ruderman \& Roberts 2002). Other density profiles have also been considered in the literature (see Soler et al. 2013, 2014). In this work three different widths of the inhomogeneous layer are considered, $l / R=[0.3,1,2]$, covering the situation of an intermediate layer $(l / R=0.3)$, a thick layer, and a fully inhomogeneous tube $(l / R=2)$. The situation with a discontinuous jump in density, $l / R=0$, is not 
considered in this work since from the numerical point of view it is the most difficult case (the jumps in some of the variables are artificially smoothed by numerical diffusion). A similar problem arises if the layer is too thin, and for this reason in the present work we have preferred to resolve well the layer and have only considered layers with $l / R \geqslant 0.3$. Nevertheless, the behavior for thin layers can be inferred from our study.

\section{MHD Equations and Initial Excitation}

The ideal three-dimensional magnetohydrodynamic (MHD) equations are numerically solved. The equations are as follows:

$$
\begin{gathered}
\frac{\partial \rho}{\partial t}+\nabla \cdot(\rho \boldsymbol{v})=0, \\
\frac{\partial \rho \boldsymbol{v}}{\partial t}+\nabla \cdot\left(\rho \boldsymbol{v} \boldsymbol{v}+P \boldsymbol{I}-\frac{\boldsymbol{B} \boldsymbol{B}}{\mu}+\frac{\boldsymbol{B}^{2}}{2 \mu}\right)=0, \\
\frac{\partial \boldsymbol{B}}{\partial t}=\nabla \times(\boldsymbol{v} \times \boldsymbol{B}), \\
\frac{\partial P}{\partial t}+\nabla \cdot(\gamma P \boldsymbol{v})=(\gamma-1) \boldsymbol{v} \cdot \nabla P,
\end{gathered}
$$

where $\boldsymbol{I}$ is the unit tensor and the rest of the symbols have their usual meaning. Temperature does not appear explicitly in the previous equations, but it is easily calculated using the ideal gas law and the values of pressure and density. Gas pressure, denoted by $P$, is included in the model mainly to avoid significant ponderomotive forces associated with nonlinear phenomena that may lead to the destruction of the tube because of the continuous accumulation of density at half the loop length (see Rankin et al. 1994; Terradas \& Ofman 2004). The plasma- $\beta$ in the present model is around 0.2 , and gas pressure is initially uniform in the whole domain.

The initial excitation is specifically chosen to deposit most of the energy in the fundamental mode along the tube axis, i.e., with longitudinal number $k_{z}=n \pi / L$ with $n=1$. For this reason, and using the results of Ruderman \& Terradas (2015), the perturbation has the following dependence:

$$
\begin{aligned}
& v_{x}=f(x, y) \cos \left(\frac{A}{B_{0} R} \frac{1}{4}(2 z-L)\right) \sin \left(\frac{\pi}{L} z\right), \\
& v_{y}=f(x, y) \sin \left(\frac{A}{B_{0} R} \frac{1}{4}(2 z-L)\right) \sin \left(\frac{\pi}{L} z\right) .
\end{aligned}
$$

Strictly speaking, there should also be a perturbation in the vertical component of the velocity, $v_{z}$. However, for the values of twist considered in this work this velocity component is rather small in comparison with $v_{x}$ and $v_{y}$, and therefore it is not included in the initial excitation.

In the absence of twist, $A=0$, the initial perturbation is in the $x$-direction only and purely sinusoidal along the $z$-coordinate. When $A \neq 0$, the polarization is in general mixed and depends on the position along the tube axis, although at $z=L / 2$ it is still in the $x$-direction. It is interesting to note that the kinetic energy (proportional to $v_{x}^{2}+v_{y}^{2}$ ) of the chosen perturbation is independent of the amount of twist, and therefore a direct comparison between different equilibria can be performed.
The shape of the perturbation in the plane $x-y$ is chosen to have a Gaussian profile centered on the tube axis,

$$
f(x, y)=v_{0} e^{-\left(x^{2}+y^{2}\right)},
$$

where we have taken $R=1$ for numerical convenience. This perturbation produces a lateral displacement of the whole loop, mostly representing the transverse $m=1$ kink oscillation when the density has a jump at the tube boundary. An important parameter in this study is the amplitude of the initial velocity, $v_{0}$, which will determine, together with some other quantities, the degree of nonlinearity of the physics in our problem.

\section{Numerical Tools \\ 4.1. MHD Simulations}

The numerical scheme used in this work to solve the timedependent MHD equations is similar to that in Terradas et al. (2016). It is an evolution of the code MoLMHD, in which a hybrid scheme is adopted. Density, pressure, and the three components of the velocity are solved with a sixth-order WENO scheme, while for the magnetic field a finite-difference scheme is used.

Although the initial equilibrium model is described in cylindrical coordinates, the nonlinear MHD equations are solved in a Cartesian coordinate system. Hence, the appropriate transformation between cylindrical and Cartesian coordinates is used. The computational domain used in the simulations is $-5<x / R<5,-5<y / R<5$, and $0<z / R<20$. Three different numerical resolutions have been considered in the present work: low resolution, with $[200,200,40]$ points in the $[x, y, z]$ coordinates; medium resolution, with $[400,400,80]$ points; and high resolution, with $[800,800,80]$ points. In the $z$-direction the resolution is lower than in the $x-y$ plane, since the oscillations have a sinusoidal profile with a typical wavelength of $2 L$. However, the presence of twist introduces additional length scales along the $z$-direction, and for this reason some simulations have been performed with an improved resolution of 320 points in the $z$-direction.

Regarding the boundary conditions, line-tying conditions are applied at the planes $z=0$ and $z=L(20 R)$, while extrapolated conditions (zero derivative) are imposed at the rest of the boundaries, allowing energy to leave the system. Note that the tube can be considered as thin since $R \ll L$.

\subsection{Analysis of Azimuthal Wavenumbers}

One of the effects of nonlinearity in the present problem is the excitation of different azimuthal wavenumbers. We have applied a local Fourier decomposition to quantify the contributions of the azimuthal numbers as a function of time. First, we have calculated the position of the center of mass (CM) of the system (at the plane $z=L / 2$, i.e., half the loop length). Since the initial perturbation (Equations (5)-(7)) mostly excites the transverse $m=1$ mode, the CM oscillates periodically with time. This magnitude has been used to define the center of our local polar coordinates, meaning that the variables of interest, in our case $v_{x}$ and $v_{y}$, have been transformed into $v_{r}$ and $v_{\phi}$. In the linear regime the position of the tube boundary is simply located at $r=R$ if we follow the motion of the tube axis. However, in the nonlinear regime, wavenumbers with $p>1$ are excited, leading to changes in the shape of the tube boundary (we use $p$ instead of $m$ to 


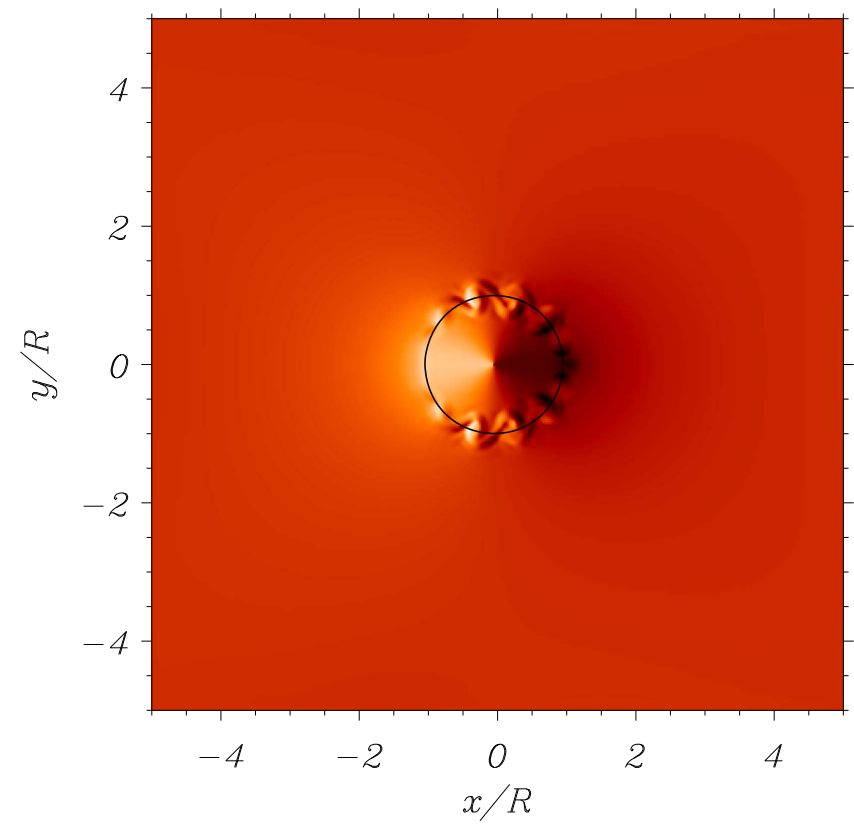

Figure 2. Example of the radial velocity calculated from the Cartesian components of the velocity using the position of the $\mathrm{CM}$ as the center of the polar coordinate system. The radial velocity is interpolated on the black circle, where the discrete Fourier transform is computed.

distinguish between the wavenumbers excited in the simulations and the eigenmode solutions). We have calculated the profile of $v_{r}$ and $v_{\phi}$ along the circular path at $r=R\left(v_{r R}\right.$ and $v_{\phi R}$ ) in the moving polar coordinate system, i.e., following the motion of the CM. An example is shown in Figure 2.

Then we have used the discrete Fourier transform to analyze the contribution of the different values of $p$ using the estimated profiles of $v_{r R}$ and $v_{\phi R}$ (namely, the function $g$ ), i.e.,

$$
G(p)=\frac{1}{N} \sum_{k=0}^{N-1} g(k) e^{-i \frac{2 \pi}{N} p k},
$$

for a discrete set of $N$ samples $(p=0, \ldots, N-1)$. In our case, the analysis is in the azimuthal direction, ranging from 0 to $2 \pi$, meaning that instead of $k$ it is more convenient to introduce the parameter $\theta_{k}=2 \pi k / N$. Therefore, with the aid of Equation (8), we know the contribution of each $p$ to the total signal, which is, using the inverse Fourier transform,

$$
g\left(\theta_{k}\right)=\sum_{p=0}^{N-1} G(p) e^{i p \theta_{k}} .
$$

Since the spectrum is symmetric about $N / 2$, we only need to consider $p=0, \ldots, N / 2$ in our analysis. Due to the parity of $v_{r R}$ and $v_{\phi R}$, the corresponding $G(p)$ is purely real and purely imaginary, respectively. In the example of Figure $2, p=1$ is dominant, but the excitation of high-order $p$ values is evident.

\section{Results for the Untwisted Tube}

We start with the magnetic configuration without twist. This will help us understand and interpret the results of the twisted magnetic tube, presented in the following sections.

In the simulations, we excite the tube by imposing the initial velocity profile given in Section 3. It is useful to know the corresponding spatial displacement in order to assess the degree of nonlinearity expected in the system. In the linear regime the relation between the tube velocity and the tube displacement is $v_{0}=\omega \xi_{0}$, where $\omega$ is the frequency of oscillation. For the transverse kink mode in the limit of $R / L \ll 1$, i.e., the thin-tube approximation, we have that $\omega_{\mathrm{k}}=k_{z} c_{\mathrm{k}}$, where $k_{z}=\pi / L$ for the fundamental mode and $c_{\mathrm{k}}$ is the kink speed. In terms of the internal Alfvén velocity we have that

$$
c_{\mathrm{k}}=\sqrt{\frac{2}{1+\rho_{e} / \rho_{i}}} v_{A i} .
$$

The relation between velocity and displacement is therefore

$$
\frac{v_{0}}{v_{A i}}=\pi \sqrt{\frac{2}{1+\rho_{e} / \rho_{i}}} \frac{\xi_{0}}{L} .
$$

For the transverse kink mode $v_{0}$ and $\xi_{0}$ can be viewed as the maximum velocity and maximum displacement of the tube axis with respect to the equilibrium position. As explained in Ruderman \& Goossens (2014), the parameter associated with the nonlinearity of the problem is indeed $\xi_{0} / R$ and not $\xi_{0} / L$. Therefore, the linear theory is valid when $\xi_{0} / R \ll 1$, i.e., when the maximum displacement of the tube is small in comparison with the radius of the tube.

Using Equation (11), we can write

$$
\frac{\xi_{0}}{R}=\frac{L}{R} \frac{v_{0}}{v_{A i}} \frac{1}{\pi} \sqrt{\frac{1+\rho_{e} / \rho_{i}}{2}},
$$

which shows that $v_{0} / v_{A i} \ll 1$ does not necessarily mean that $\xi_{0} / R \ll 1$. This magnitude depends also on the ratio $L / R$, which is in general quite large, at least for the observed oscillating loops in the solar corona. Hereafter, we perform a parametric study changing the main parameter of our problem, $\xi_{0} / R$.

\subsection{Linear Results}

As a method of testing the simulations and numerical method, we start our study with the analysis of the evolution of the system for a perturbation in the linear regime, $\xi_{0} / R=0.05 \ll 1$. We perform the analysis of the azimuthal wavenumbers explained in Section 4.2 on the output of the numerical simulations. The results are plotted in Figure 3. We obtain the expected behavior: the tube is oscillating laterally around the equilibrium position at the kink frequency $\left(\omega_{\mathrm{k}}\right)$. The sinusoidal motion of the tube axis is slightly attenuated with time (see dashed line in the top panel), and it is due to the process of continuum damping associated with the presence of the inhomogeneous layer between the core of the tube and the external medium (see the time-dependent analysis in Terradas et al. 2006). At the same time the amplitude of the azimuthal velocity component grows with time (see bottom panel) owing to the energy that is pumped into the layer. The increase in the amplitude of the azimuthal velocity component is bounded since the initial perturbation has a finite energy. The attenuation at the end of the time interval is due to numerical dissipation.

The agreement between the results of the simulation and the theoretical predictions is remarkable. The exponential decay given by the analytical expressions for the damping rate works very well after two or three periods, while at the beginning of the evolution it is better fitted by a Gaussian profile (Pascoe et al. 2012; Hood et al. 2013; Ruderman \& Terradas 2013). 

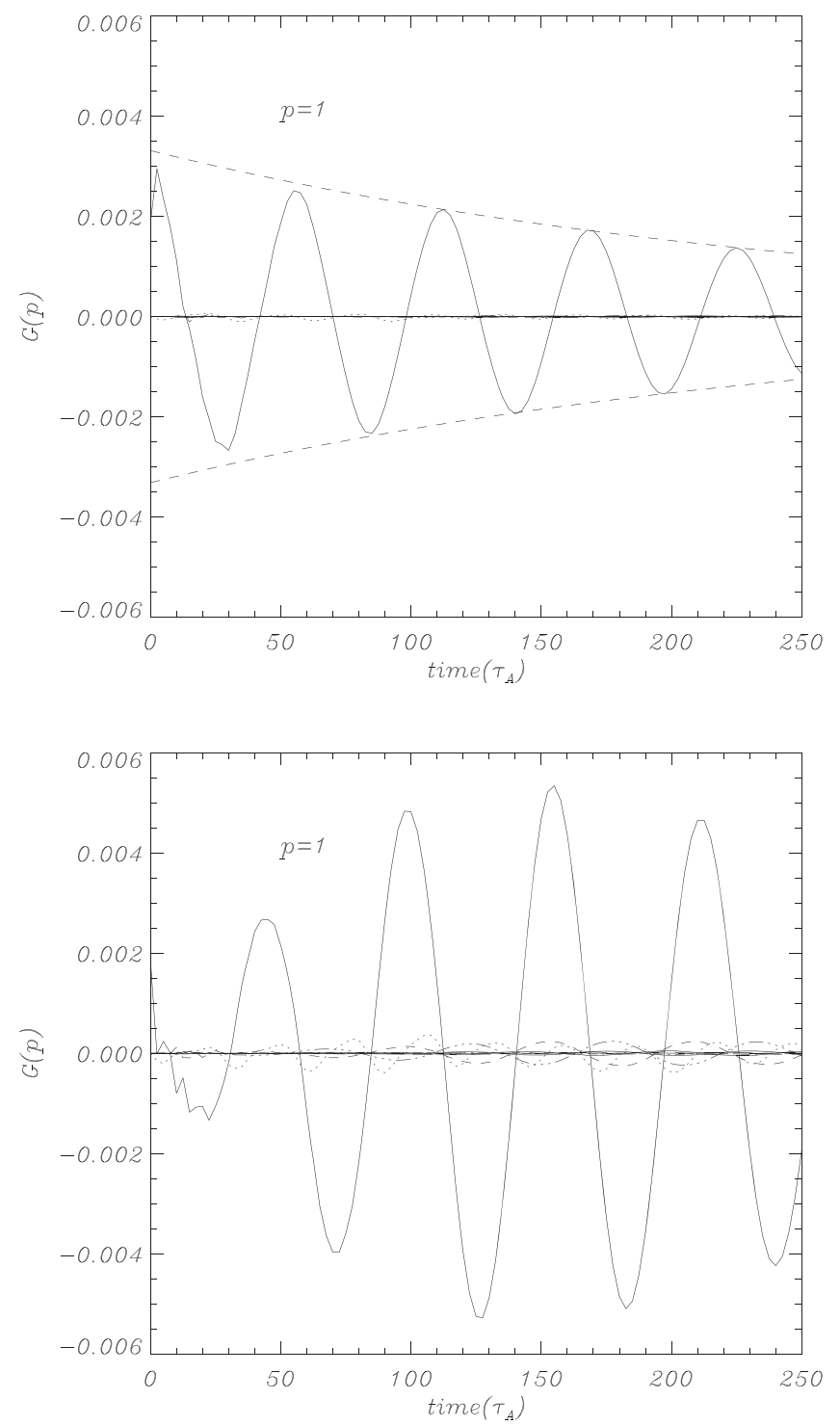

Figure 3. Fourier coefficients as a function of time for the radial velocity $v_{r R}$ (top panel) and azimuthal velocity $v_{\phi R}$ (bottom panel). The contribution of the $p=1$ is dominant. Higher values of $p$ (plotted with dotted and dashed lines) are due to weakly nonlinear effects and are clearer in the bottom panel. In the top panel, an exponential fit using the last three peaks has been performed to estimate the damping time. In this case, $l / R=0.3$ and there is no twist.

From the simulations we infer a damping per period of 4.3, while the analytical theory provides a value of 4.1 (see the analytic expressions for the thin-tube and thin-boundary approximations in, e.g., Ruderman \& Roberts 2002). The good match we obtain in our runs is similar to that of Magyar \& Van Doorsselaere (2016) in the linear regime. From the bottom panel of Figure 3 we appreciate a very weak excitation of higher $p$ values, which is related to nonlinear effects described in detail in the following sections.

\subsubsection{Effect of Numerical Resolution}

An increase in the grid resolution mostly affects the small scales that are generated in the inhomogeneous layer, while the attenuation times of the oscillations are essentially unaltered. This has been checked comparing the results for medium and high resolutions. This means that the dissipation scales are much shorter than the global characteristic scale of the transverse kink motion. This behavior agrees with the results of the quasi-mode calculations that indicate that for small dissipation coefficients (resistivity or viscosity) the damping times are independent of the amount of dissipation in the system (e.g., Poedts \& Kerner 1991; Van Doorsselaere et al. 2004; Terradas et al. 2006; Soler et al. 2013). An increase in the resolution reduces the numerical dissipation in the azimuthal velocity component found at the end of the time series in the bottom panel of Figure 3.

\subsection{Nonlinear Results}

Now we concentrate on a weakly nonlinear situation $\left(\xi_{0} / R=0.2\right)$. It was shown by Terradas et al. (2008), Antolin et al. (2014, 2015, 2017), Magyar et al. (2015), and Magyar \& Van Doorsselaere (2016) that under such conditions the tube boundary develops deformations and changes associated with the KHI. For a proper quantification of the deformation of the tube boundary, we perform again the analysis of the azimuthal wavenumbers. The time evolution of the Fourier coefficients is plotted in Figure 4 (top panel). The value $p=1$ has the largest amplitude since the initial perturbation is chosen to precisely excite this mode and shows again attenuation with time. But now $p=2$ has a significant amplitude and is excited from the beginning of the time evolution. The excitation of higher-order $p$ values is also present in the top panel of Figure 4, but only for times larger than $t / \tau_{\mathrm{A}}=150$.

The value of $p=2$ has a period that is essentially half of that of $p=1$, as can be appreciated in Figure 4. This nicely agrees with the analytical results of Ruderman \& Goossens (2014), predicting a nonlinear coupling between $p=1$ and $p=2$ (see also Ruderman et al. 2010, for the case of propagating waves instead of standing). These authors found that the frequency (period) of the mode $p=2$ is twice (half) the frequency (period) of the $p=1$, which acts as a driver of $p=2$ (see their Equations (82) and (83); see also the recently accepted paper of Ruderman 2017), which is confirmed in our simulation. Antolin et al. (2017) have also described this effect in their numerical analysis of transverse kink oscillations. From the physical point of view, the deformation of the edge of the tube associated with $p=2$ can be understood if we concentrate on the plane at half the tube length at the early stages of the time evolution; see Figure 5. At $t=0$ the tube with a cylindrical shape is located at $x=0$, and the perturbation produces the lateral displacement of the whole tube, which immediately starts to decelerate because of the effect of the line-tied magnetic field lines $(p=1)$. The front of the tube decelerates slightly faster than the rear since at the front the magnetic lines are more curved, the magnetic tension is stronger, and this produces a decrease of the width of the tube along the $x$-direction. At the same time the width along the $y$-direction increases because the motions are essentially incompressible, meaning that the area of the cross section has to remain constant. Once the tube has reached its maximum displacement, the process is reverted. The width of the tube increases in the $x$-direction and reaches the maximum elongation at the initial position of the equilibrium. The motion of the tube continues in the negative $x$-direction, and the width of the tube decreases in the horizontal direction and increases in the vertical direction, recovering the situation found at the maximum positive displacement, but now the tube is located at the maximum displacement in the negative $x$-direction. For this reason, the period of the deformation associated with 

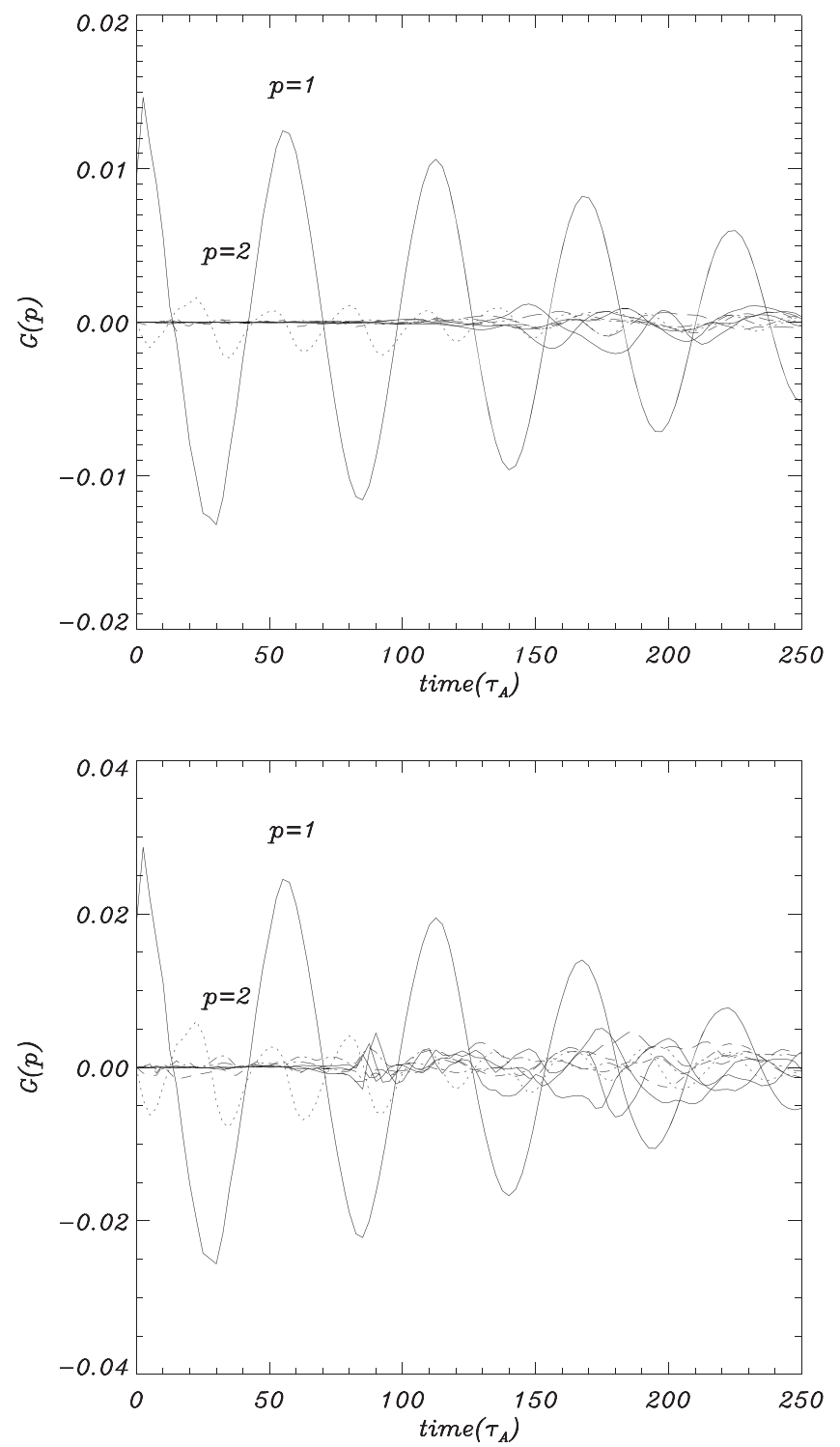

Figure 4. Fourier coefficients as a function of time for the radial velocity $v_{r R}$. The contribution of $p=1$ is dominant. Higher values of $p$ are due to nonlinear effects. The value of $p=2$ is oscillating at half the period of $p=1$. In this case $l / R=0.3$ and there is no twist. In the top panel the amplitude of the initial excitation $\left(\xi_{0} / R=0.2\right)$ is half the amplitude in the bottom panel $\left(\xi_{0} / R=0.4\right)$. Note the different ranges in the vertical axis of the plots.

$p=2$ has half the period of the transverse $p=1$ displacement. These explanations are based on the fact that changes in the vertical direction of the velocity (z-component), perpendicular to the plane of reference, produce a negligible contribution to the total divergence of the velocity, meaning that if this magnitude is basically zero then contractions/expansions in the $x$-direction have to be balanced by equal expansions/ contractions in the $y$-direction.

Interestingly, the excitation of higher-order $p$ values ( $p$ greater than 2) in the top panel of Figure 4 is evident for $t / \tau_{\mathrm{A}}>150$. Contrary to the damped behavior of modes $p=1$ and $p=2$, their amplitude in general increases with time. In order to quantify this behavior, we have performed a sum of Fourier coefficients. In particular, we have calculated $\sum_{p=3}^{N / 2}|G(p)|$, which is represented in Figure 6 (see dashed line) as a function of time. The increase with time of this magnitude is evident up to times around $t / \tau_{\mathrm{A}}=200$. This behavior is due to the
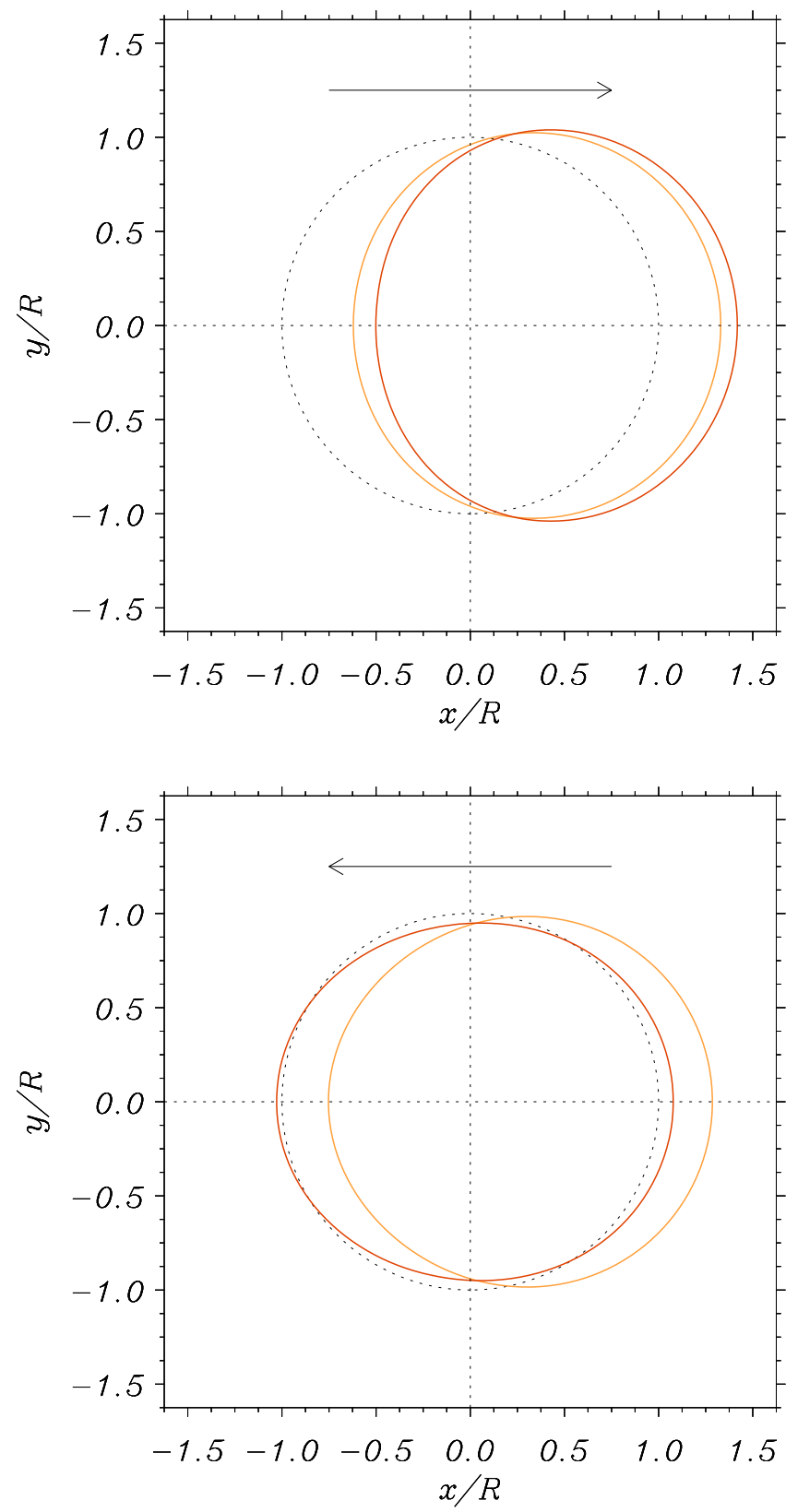

Figure 5. Shape of the tube cross section at different times. In the top panel, the tube is moving in the positive $x$-direction. In the bottom panel, the motion is in the negative $x$-direction. The excitation of the $p=2$ mode, driven by the $p=1$ mode, is clear in this plot. The circle plotted with dots represents the shape of the tube at $t=0$.

development of the KHI at the shear layer. The density distribution at a given time instant is represented in the top panel of Figure 7. In this plot the still weak deformations of the tube boundary are due to the early stages of the development of the KHI.

We have repeated the run but with an initial amplitude that is twice the one in the previous simulation. The results for $\xi_{0} / R=0.4$ are represented in the bottom panel of Figure 4 . Now the amplitude of the $p=2$ mode with respect to that of the $p=1$ mode is larger in comparison with the top panel of Figure 4. In fact, the theoretical prediction of Ruderman \& Goossens (2014) about the quadratic dependence between the amplitude of $p=1$ and $p=2$ is also found in the simulations, and this can be inferred from the comparison of the top and 


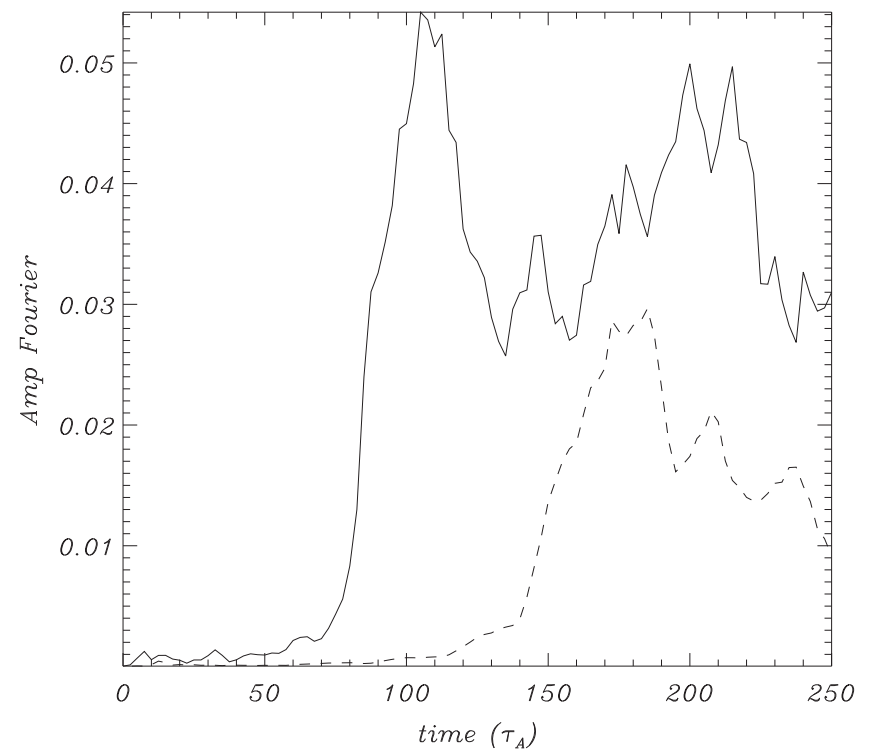

Figure 6. Sum of Fourier coefficients, $\sum_{p=3}^{N / 2}|G(p)|$, as a function of time for the radial velocity $v_{r R}$. The dashed line corresponds to an initial amplitude of $\xi_{0} / R=0.2$, which is half of that of the solid line, $\xi_{0} / R=0.4$. In this case, $l / R=0.3$ and there is no twist.

bottom panels of Figure 4 (the ratio of the amplitudes $p=1$ to $p=2$ in the top panel with respect to the bottom panel, where the initial amplitude has been doubled, is about 4). The increase of amplitude of the nonlinear excited high-order $p$ values is also evident in this plot. The evolution of the sum of Fourier coefficients is plotted in Figure 6 (solid line). The onset of the $\mathrm{KHI}$ is produced at earlier stages (around $t / \tau_{\mathrm{A}}=80$ ), and the rise of the instability is faster. The amplitude represented in Figure 6 for the nonlinear case is larger than that for the weakly nonlinear situation, meaning that more energy is eventually deposited in high-order $p$ values than in the first case. The density distribution at a given time instant is represented in the bottom panel of Figure 7, where significant deformations of the boundary are visible owing to the excitation of $p>2$ (compare with top panel).

An additional conclusion from the comparison of the results in Figure 4 is that the attenuation with time of the $p=1$ mode is faster for the largest initial amplitude of excitation. In Figure 8 the two attenuation profiles for $p=1$ have been superimposed by choosing a proper normalization factor. The plot indicates that, for times shorter than $t / \tau_{\mathrm{A}}=80$, the two curves are almost identical. This means that although the coupling with the $p=2$ is stronger for the largest amplitude of excitation, this does not significantly affect the damping. Nevertheless, the differences in the two curves start to be clear when the KHI develops, i.e., for $t / \tau_{\mathrm{A}}>80$. Therefore, the development of the KHI instability enhances the attenuation of the oscillation since part of the energy is transferred from the initially organized flow of the $p=1$ mode to higher-order $p$ values. This is in agreement with the results of Magyar \& Van Doorsselaere (2016).

\subsubsection{Analytical Estimation of KHI Onset Times}

The onset time for the instability in the untwisted case can be inferred by applying the results obtained by Browning \& Priest
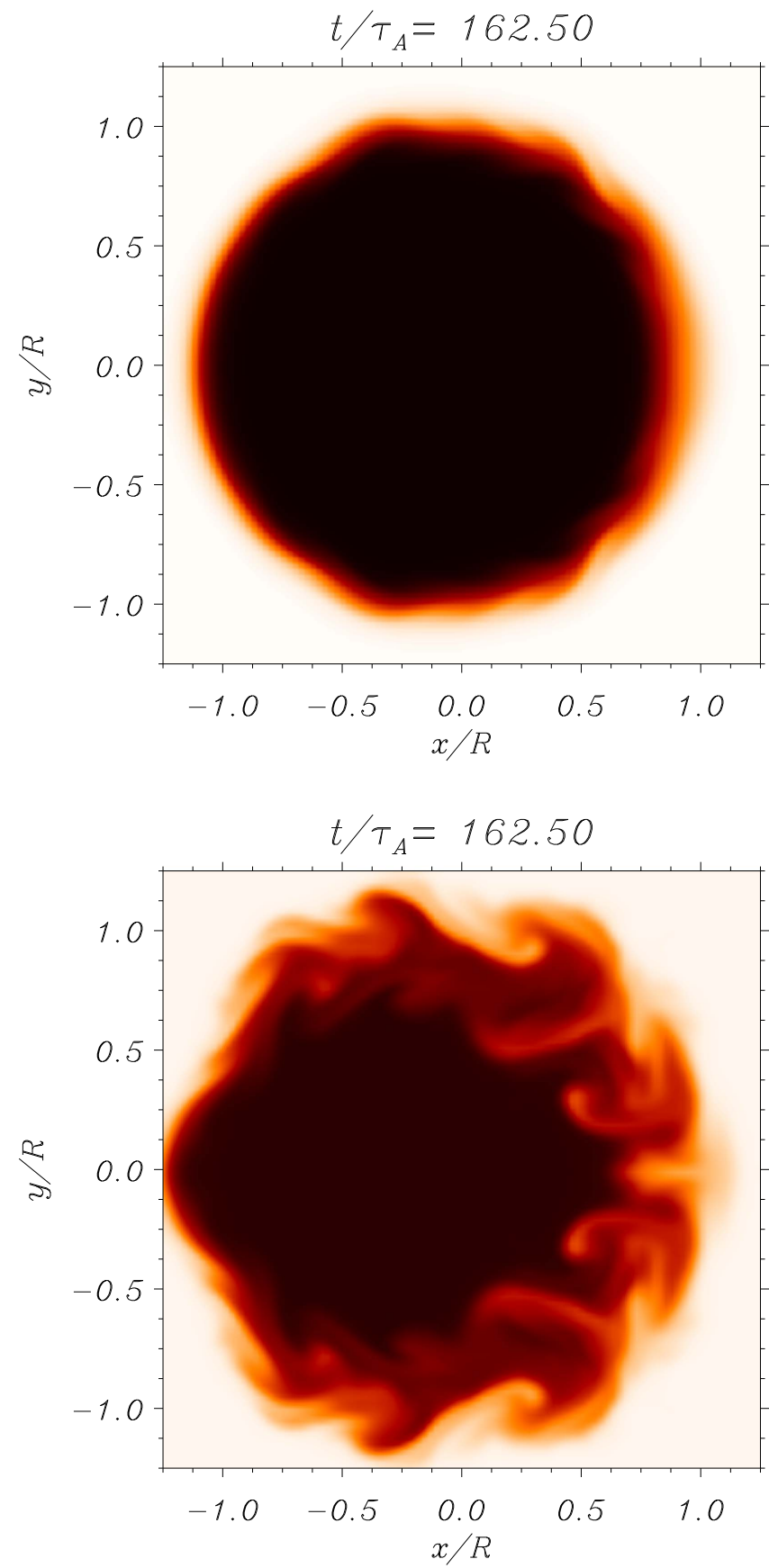

Figure 7. Snapshot of the $2 \mathrm{D}$ density distribution at half the tube length $(z=L / 2)$ at a given time. In the top panel the amplitude of the initial excitation $\left(\xi_{0} / R=0.2\right)$ is half the amplitude in the bottom panel $\left(\xi_{0} / R=0.4\right)$.

(1984) in a strong phase-mixing situation. Some caution is needed here since, strictly speaking, the results of Browning \& Priest (1984) are applicable for $m=0$ only.

Allan \& Wright (1997) defined the following normalized maximum growth rate based on Browning \& Priest (1984):

$$
\Gamma_{\mathrm{KH}} \approx \frac{1.7 V_{0}}{4 \omega_{\mathrm{A}} L_{\mathrm{ph}}},
$$

where $V_{0}$ is the wave amplitude, $\omega_{\mathrm{A}}$ the frequency at the resonance, and $L_{\mathrm{ph}}$ the phase-mixing length. The phase-mixing 


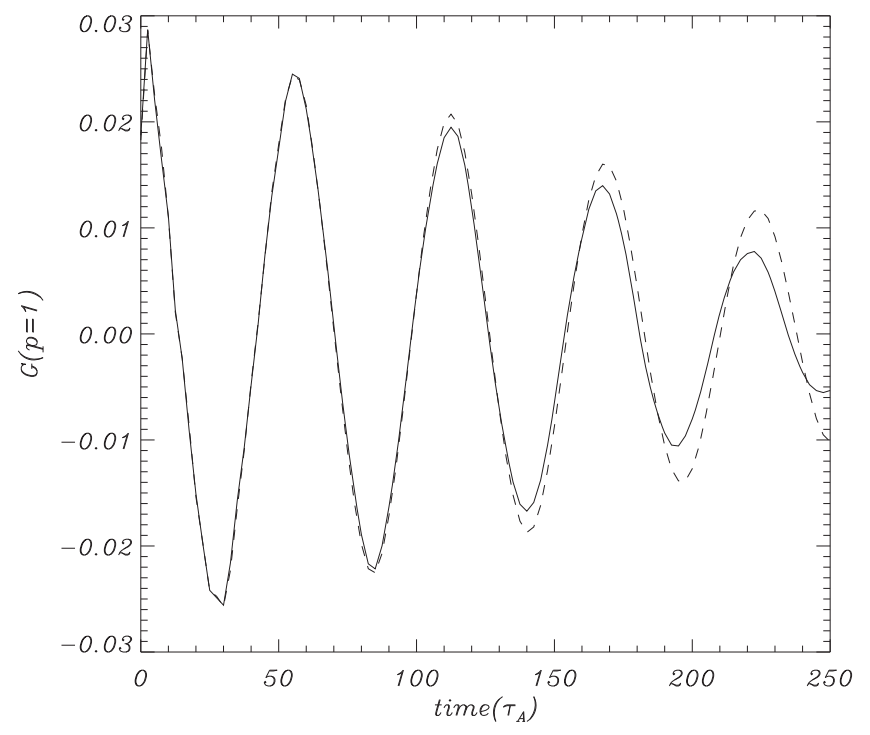

Figure 8. Fourier coefficients as a function of time for the radial velocity $v_{r R}$ $(p=1)$ corresponding to two different amplitudes of excitation (same as in Figure 4). The dashed line corresponds to an amplitude that is half of the black line and has been normalized for comparison purposes. The attenuation is faster when the amplitude is larger.

length is defined as (see Mann et al. 1995; Wright \& Rickard 1995)

$$
L_{\mathrm{ph}}=\frac{2 \pi}{d \omega_{\mathrm{A}} / d r t} .
$$

In our model, and in the linear regime without twist, the dependence with position of the Alfvén frequency is known since we know that the density profile is sinusoidal and the magnetic field is constant; calculating the derivative of the Alfvén frequency, we find that $L_{\mathrm{ph}} \approx l /(\Omega t)$, where $l$ is the width of the inhomogeneous layer and $\Omega$ is a characteristic frequency. This magnitude is time dependent, decreasing as $t^{-1}$, because the phase-mixing process continuously generates smaller length scales with time. The amplitude $V_{0}$ at the resonant layer depends also on time since energy is gradually pumped into the layer until all the energy of the initial perturbation is deposited there. This means that $\Gamma_{\mathrm{KH}}$ is also a function of time. Allan \& Wright (1997) defined the normalized growth rate of the instability in such a way that the growth of the KHI during a quarter cycle of a large velocity shear will be significant if $\Gamma_{\mathrm{KH}}$ is comparable to or greater than unity. Using the results of the linear simulations, we compute $\Gamma_{\mathrm{KH}}$, which is linearly proportional to the amplitude of the velocity shear at the resonant position. If nonlinearity does not significantly modify the rate at which the energy is pumped into the layer, then we can use the profile of $\Gamma_{\mathrm{KH}}$ in the linear regime, namely, for very small amplitudes of oscillation, to infer the onset time for the instability in the nonlinear regime, i.e., when the amplitudes are larger. We only need to multiply $\Gamma_{\mathrm{KH}}$ by the corresponding ratio of amplitudes. An example is represented in Figure 9, for a value of $t / \tau_{\mathrm{A}} \approx 175$, i.e., when the instability develops for the case $\xi_{0} / R=0.2$, the value of $\Gamma_{\mathrm{KH}}$ is around 1.5. Now we can use this value to infer the onset time for other amplitudes. For the case $\xi_{0} / R=0.4$ the curve $\Gamma_{\mathrm{KH}}$ intersects 1.5 (not shown here) at $t / \tau_{\mathrm{A}} \approx 90$, which is in

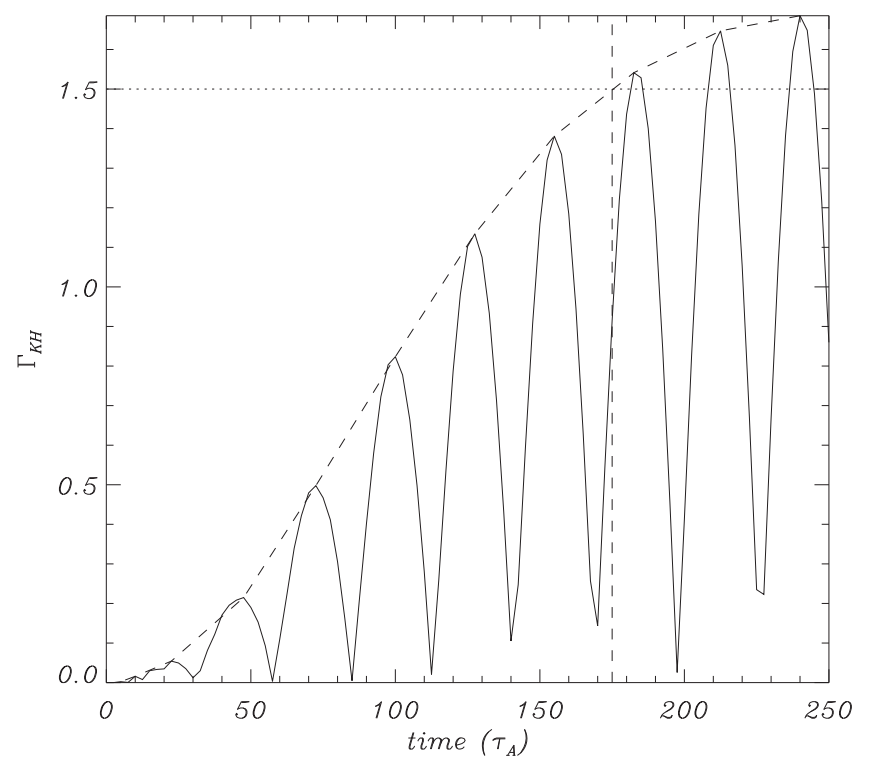

Figure 9. Normalized maximum growth rate as a function of time for $\xi_{0} / R=0.2$ based on the linear results and Equation (13).

agreement with the results of the nonlinear simulations (see Figure 6). Therefore, Equation (13) can be used as a crude estimate for the onset times of the KHI instability.

Terradas et al. (2016) have used this assumption to estimate the onset times of the instability in a curved flux rope configuration with an embedded prominence and have found that slightly lower values for $\Gamma_{\mathrm{KH}}$ (around 0.6) are required to have a good match with the simulations.

\subsubsection{Effect of Numerical Resolution}

Contrary to the situation in the linear regime, in the nonlinear case the effect of the mesh resolution is more determinant. The growth rates of the unstable $\mathrm{KH}$ modes have a strong dependence on the azimuthal wavelengths of the modes. Therefore, it is expected that the shorter the azimuthal wavelength resolved by the grid, the faster the onset of the instability. This is true when the results for low and medium resolution are compared (see Figure 10). Nevertheless, for medium- and high-resolution simulations, the times for the onset of the instability are very similar, indicating convergence of the results. We have also performed a simulation with $[400,400,320]$ points, i.e., with better resolution in the $z$-direction, and have arrived to the same conclusion. Hence, we are sure that the numerical simulations are not significantly affected by the number of mesh points as long as we use medium or high resolutions. The same conclusion applies to the attenuation profiles.

\section{Results for the Twisted Tube}

The effect of magnetic twist on standing transverse oscillations is investigated in this section. It is important to mention that the initial velocity perturbation, which depends on the amount of twist, is crucial to avoid the excitation of longitudinal harmonics. For example, an initial perturbation with $n=1 \quad\left(k_{z}=n \pi / L\right)$ in $v_{x}$ and zero in $v_{y}$ significantly excites the longitudinal mode $n=3$ in the case of a twisted tube. The particular perturbation given in Section 3 allows us to concentrate on the fundamental mode $(n=1)$ and proceed as in the previous section. 


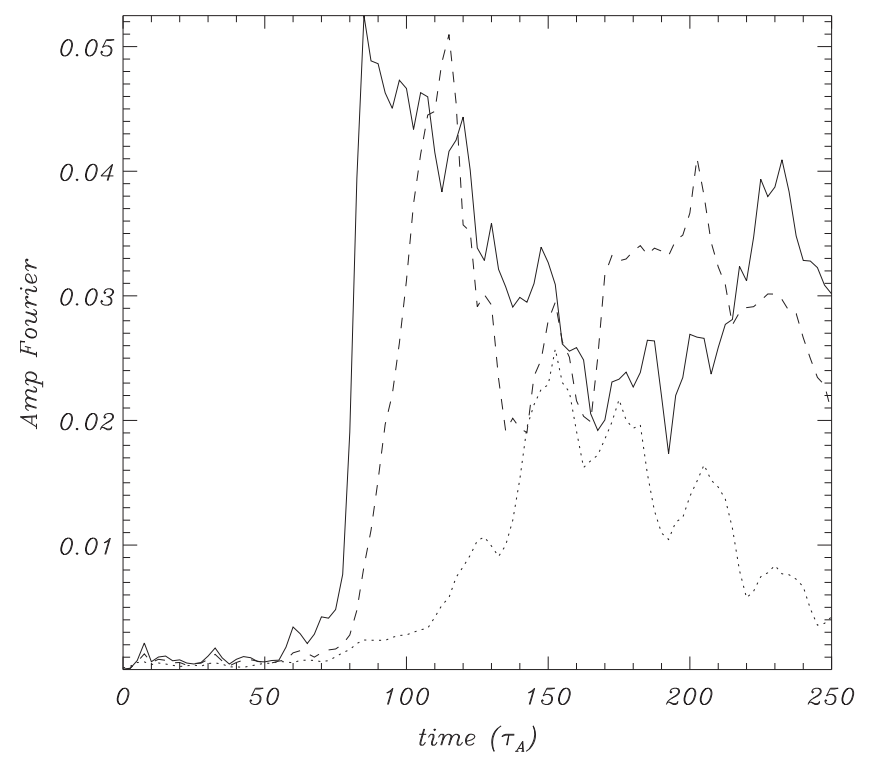

Figure 10. Sum of Fourier coefficients, $\sum_{p=3}^{N / 2}|G(p)|$, as a function of time for the radial velocity $v_{r R}$. The solid, dashed, and dotted lines correspond to the situation of high, medium, and low resolution, respectively. In this case, $l / R=0.3$

\subsection{Linear Results}

In the linear regime the simulations show that the frequency of the standing kink oscillation is essentially unaltered, meaning that $\omega=\omega_{\mathrm{k}}$. This is in agreement with the analytical results of Ruderman \& Terradas (2015) in the situation of weak twist and for exactly the same magnetic twist model (see also Ruderman 2007, for other twist profiles). The effect of twist on propagating waves is much more significant, for example, regarding the change in the kink frequency, e.g., Terradas \& Goossens (2012) and Ruderman (2015).

For standing waves the polarization of the motions along the loop axis changes according to the profile derived by Ruderman \& Terradas (2015) and given by Equations (5)-(6) (see also Terradas \& Goossens 2012). Regarding the attenuation associated with the inhomogeneous layer plus magnetic twist, so far there are no studies about the damping times for standing waves in such a model, although some work has been done for propagating waves (see Karami \& Bahari 2010). A detailed eigenmode analysis is required, but it is beyond the scope of this work since the main aim here is the KHI. It suffices to mention that from the time-dependent simulations we do not find a significant change in the efficiency of the resonant damping mechanism for the values of twist considered in this work.

In Figure 11 (top panel) an example of the time evolution of the Fourier coefficients is displayed. Again $p=1$ is dominant and the excitation of $p=2$ is extremely weak since we are in the linear regime. In this situation, an increase on the grid resolution has the same effect as in the nontwisted loop. The damping times are not affected, but short spatial scales, taking place around the resonant positions, are resolved better. In Figure 11 (bottom panel) we find again the increase of amplitude of the azimuthal component associated with the resonant damping. In this plot $p=2$ can be also identified.

It is worth mentioning that, in the case of a twisted magnetic tube, it is more convenient to use, instead of the azimuthal velocity component, $v_{\phi}$, the perpendicular component to the
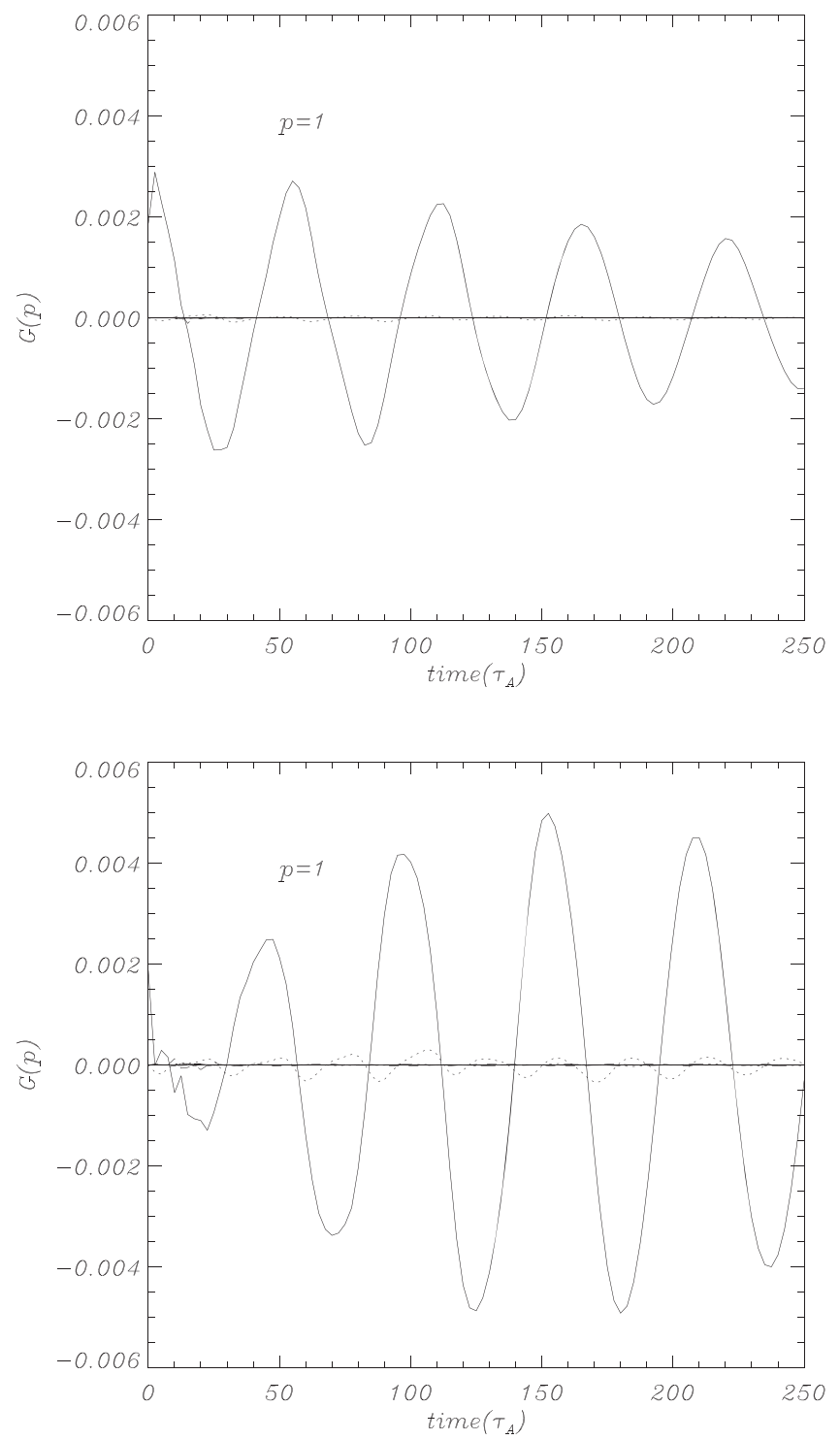

Figure 11. Fourier coefficients as a function of time for the radial velocity $v_{r R}$ (top panel) and azimuthal velocity $v_{\phi R}$ (bottom panel) in the linear regime. The contribution of $p=1$ is dominant. In this case, $l / R=0.3$ and $B_{\phi} / B_{z}=0.2$.

magnetic field lying on magnetic surfaces (still cylindrical) and defined as $v_{\perp}=\left(B_{z} v_{\phi}-B_{\phi} v_{z}\right) / B$. Twist introduces a vertical component of the velocity, $v_{z}$, which in our case has other contributions due to gas pressure and ponderomotive forces. It is also appropriate to use the parallel component of the velocity to the magnetic field lines, $v_{\|}=\left(B_{\phi} v_{\phi}+B_{z} v_{z}\right) / B$. This component of the velocity is strictly zero only when the plasma- $\beta$ is zero and when the linearized MHD equations are considered.

An example of the shear in $v_{\perp}$ and in the linear regime is plotted in Figure 12 across the inhomogeneous layer. For comparison purposes the result with magnetic twist (dashed line) is plotted together with the simulation without twist (solid line). The peak around $y / R=1$ is due to the energy transference between the global motion of the tube and the Alfvénic localized oscillations. The spatial scales in the layer decrease with time, and consequently the shear increases (compare top and bottom panels) owing to the phase-mixing process. Interestingly, the results shown in Figure 12 reveal that the motions for the tube with magnetic twist seem to have less 

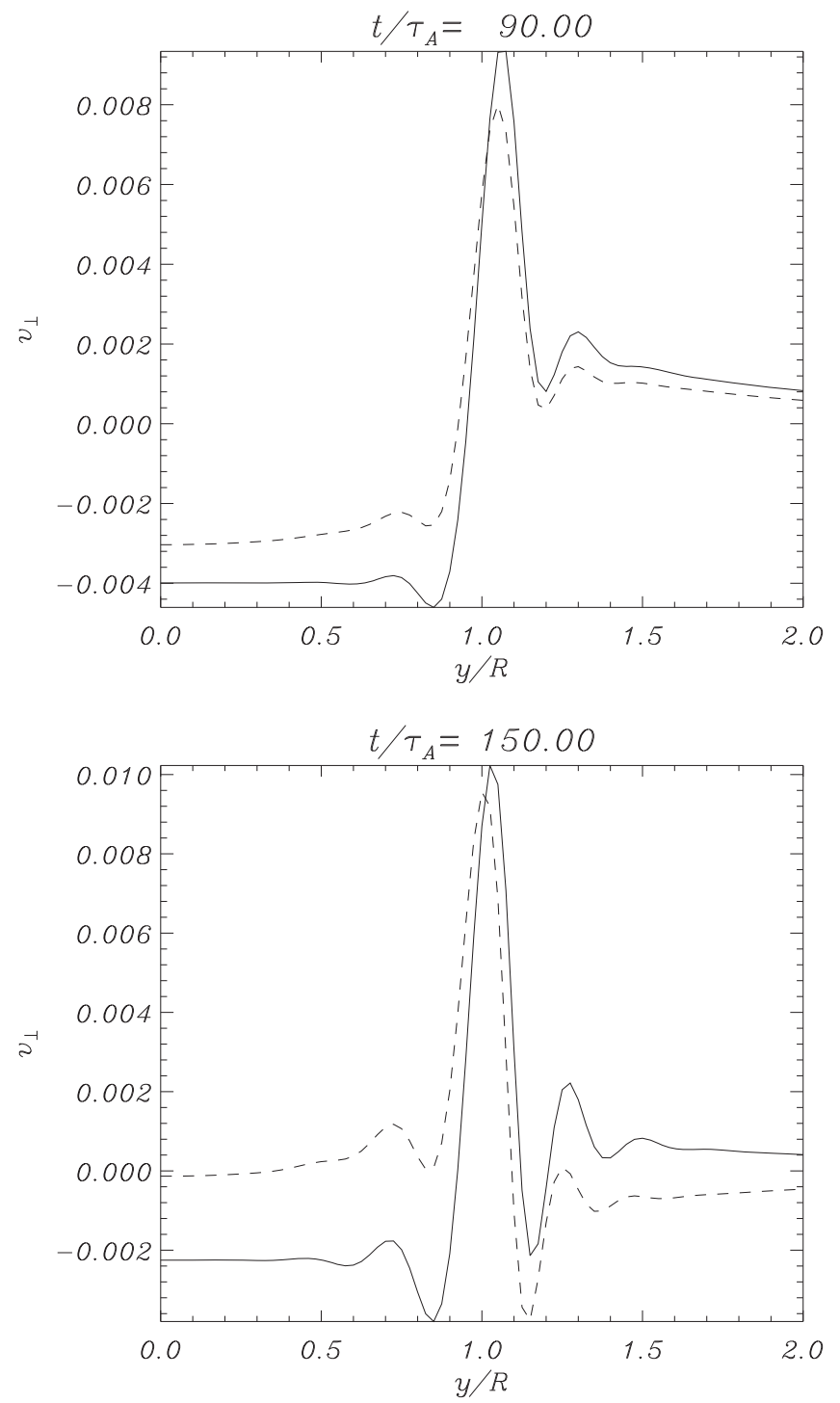

Figure 12. Perpendicular velocity component to the magnetic field, $v_{\perp}$, as a function of position (in a range between the center of the tube and $2 R$ ) at two different times. The solid line corresponds to the case without twist, while the dashed line corresponds to the situation $B_{\phi} / B_{z}=0.4$. In these simulations, $l / R=0.3$ and the linear regime is considered.

shear than the motions for the untwisted tube. To properly quantify this effect, we have computed the shear vorticity, defined as the derivative of $v_{\perp}$ along the $y$-direction. The idea is to integrate this magnitude in space $(0<y / R<2)$, but taking its absolute value to avoid effects due to changes in sign. This provides a proxy for the total shear associated with the oscillations of the tube. The integrated shear vorticity (ISV) is plotted in Figure 13 as a function of time. The interpretation is the following: at $t=0$ the ISV is essentially the same for the untwisted and twisted tubes and starts to fluctuate owing to the kink motion. Before completing a single period, the ISV increases owing to the generation of small scales and the rise in $v_{\perp}$ because energy is pumped into the layer. Then it reaches a saturation point where numerical dissipation prevents a further decrease of the spatial scales. But the most relevant information that we can extract from Figure 13 is that, in the case of a twisted tube, the ISV is, after a short transient, always below the value for the untwisted tube and its growth rate is smaller. This means that one of the effects of twist is to reduce the total

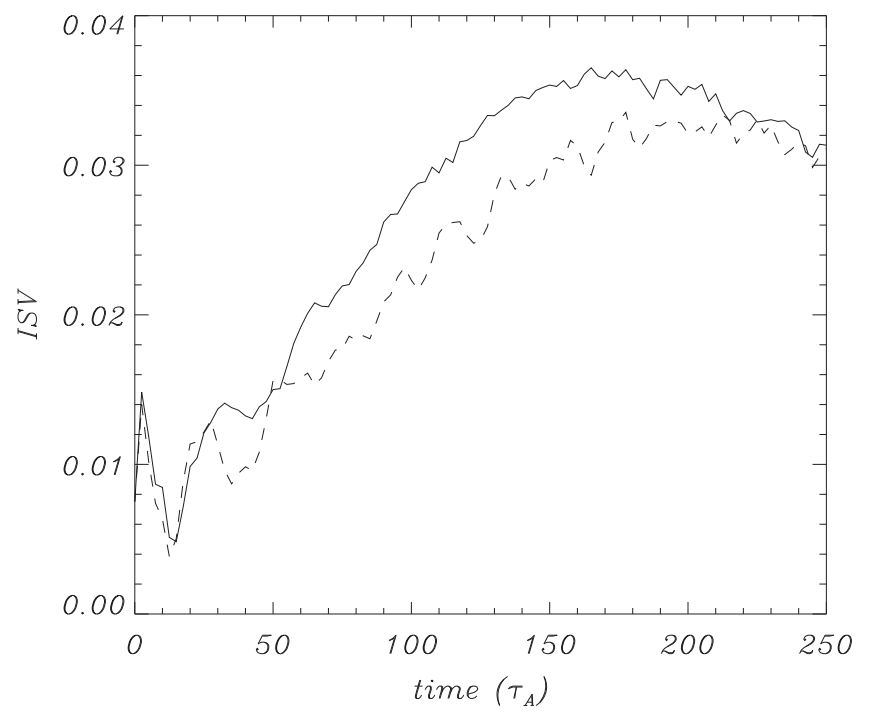

Figure 13. ISV as a function of time. The solid line corresponds to the case without twist, while the dashed line represents the situation for $B_{\phi} / B_{z}=0.4$.

shear velocity at the tube boundary with respect to the oscillations in the untwisted tube. As we show in the following sections, this has important consequences regarding the development of the KHI.

\subsection{Nonlinear Results}

In the nonlinear regime $\left(\xi_{0} / R=0.4\right)$ and under the presence of twist, we again find the development of the KHI. However, now the azimuthal component of the magnetic field changes the properties of the oscillation. Since the polarization of the motion varies along the tube, the shear flow is less organized in the twisted case. The shear flow profile due to $v_{\perp}$ at the tube boundary is still responsible for the instability, and by definition this velocity component is perpendicular to the equilibrium magnetic field. Therefore, the equilibrium magnetic field should not have the expected stabilizing effect of the situation when there is a magnetic component along the shear flow (e.g., Chandrasekhar 1961). Nevertheless, twist changes the properties of the transverse oscillation, as we have already seen in the linear regime, and has an effect on the instability onset.

To facilitate the comparison of the results, we proceed as in Section 5.2 and concentrate on the plane at $z=L / 2$. We have calculated the Fourier coefficients for two different values of twist $\left(B_{\phi} / B_{z}=0.2\right.$ and 0.4$)$, which are plotted in Figure 14. We can compare these plots with the results for the same situation without twist, represented in bottom panel of Figure 4. The coupling with $p=2$ is still present, and the amplitude of this mode does not seem to depend strongly on the amount of twist. Nevertheless, the main differences between the plots are in the timing for the onset of the excitation of higher-order values of $p$. Further evidence of this effect is found in the density distribution for the two twisted tube models at the same instant, plotted in Figure 15. The deformations of the boundary are less pronounced in the case of $B_{\phi} / B_{z}=0.4$ in comparison with the situation $B_{\phi} / B_{z}=0.2$. These plots can also be compared with the untwisted tube, shown in the bottom panel of Figure 7, displaying a much more complex structure in the azimuthal direction. It is clear that magnetic twist delays the appearance of the KHI instability. However, we have to bear in mind that 

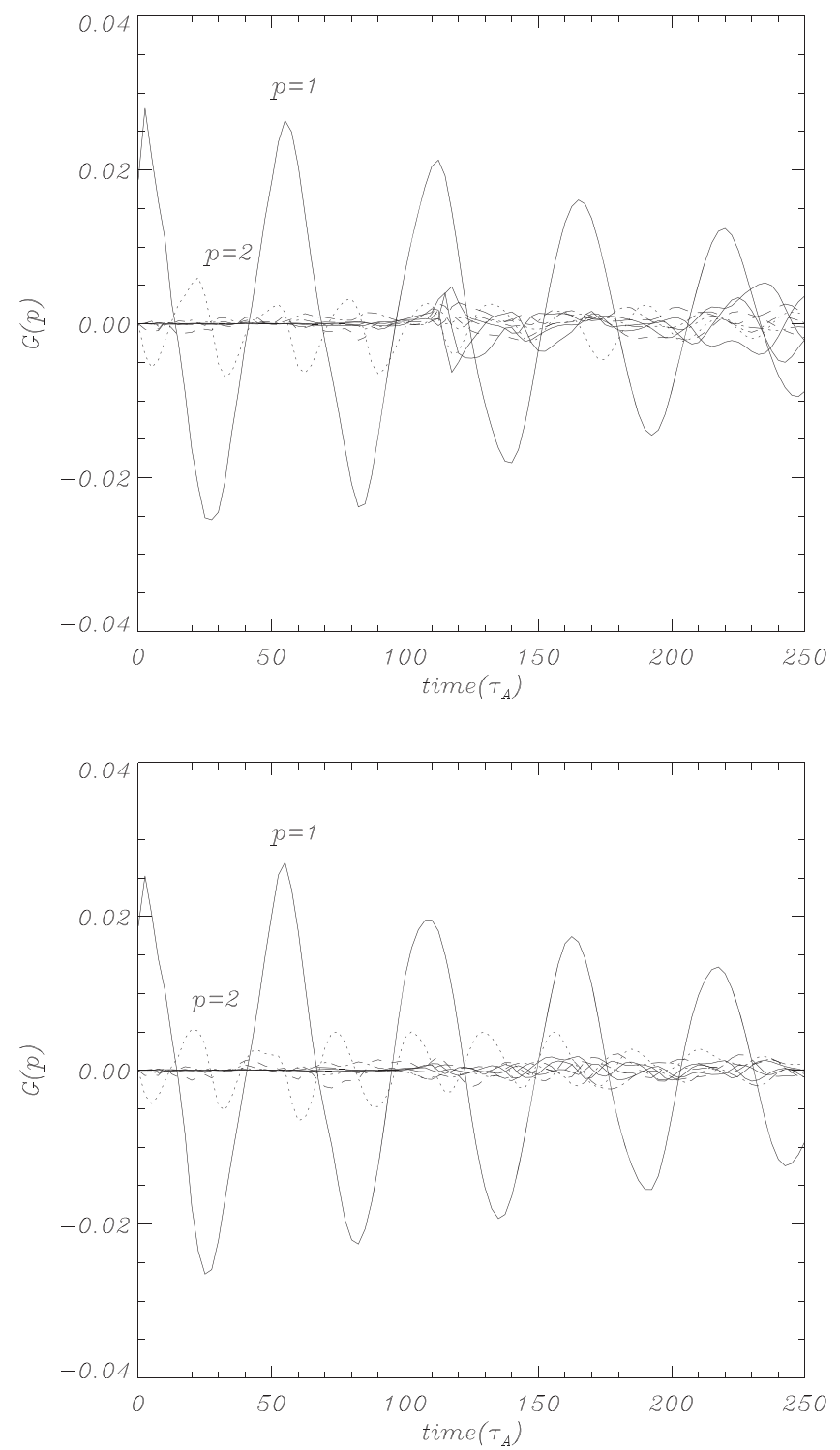

Figure 14. Fourier coefficients as a function of time for the radial velocity $v_{r R}$ The contribution of $p=1$ is dominant. Higher-order $p$ values are due to nonlinear effects. The $p=2$ is oscillating at half the period of $p=1$. In this case, $l / R=0.3$. In the top panel $B_{\phi} / B_{z}=0.2$, while in the bottom panel $B_{\phi} / B_{z}=0.4$. The excitation is nonlinear $\left(\xi_{0} / R=0.4\right)$.

the helical vortices introduce structuring along the $z$-direction and that these vortices may end up colliding with each other owing to the different growth rates depending on the direction of swing. This can significantly change the structuring in the azimuthal direction at more evolved stages of the system.

In Figure 16 the contribution of high-order $p$ values is plotted as a function of time for different values of twist. The stronger the azimuthal component of the field, the later the development of the KHI. From Figure 16, we have estimated the onset time for the instability, $\tau_{\mathrm{KHI}}$, according to a given amplitude threshold, in this case 0.01. The results, shown in Figure 17, indicate that the slope of the curve increases when twist is augmented. A twisted tube with $B_{\phi} / B_{z}=0.4$ and $l / R=0.3$ has a typical onset time for the instability around three times larger than the untwisted tube.

So far, we have concentrated on a given width of the inhomogeneous layer $(l / R=0.3)$, but it is also interesting to repeat the simulations changing $l / R$ to understand how the
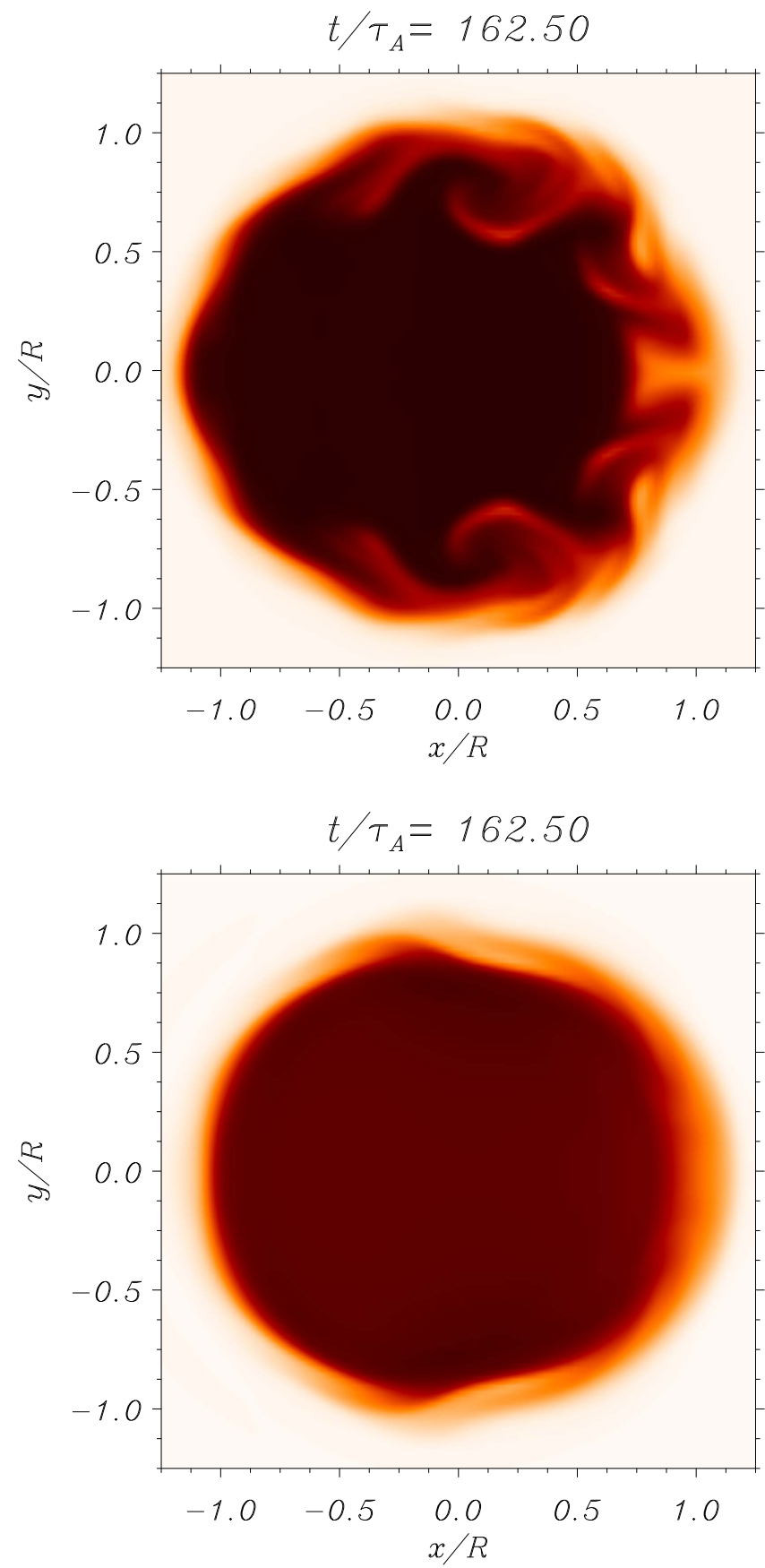

Figure 15. Snapshot of the two-dimensional density distribution at half the tube length $(z=L / 2)$ at a given time. In the top panel $B_{\phi} / B_{z}=0.2$, while in the bottom panel $B_{\phi} / B_{z}=0.4$. The development of the KHI is delayed when magnetic twist is increased. For these simulations, $l / R=0.3$.

onset times of the instability are modified. The results are also plotted in Figure 17. We find that, as the thickness of the layer is increased, the instability appears at later times; see the density distribution of Figure 18 for a particular case. This behavior is already found in the untwisted tube. The interpretation is related to the generation of the spatial scales at the inhomogeneous layer owing to the phase-mixing process. Since the generation of the velocity shear scales as $L_{\mathrm{ph}} \approx l /(\Omega t)$, i.e., approximately linear in $l$, it means that for thick layers the time required to achieve a given spatial scale is always larger than for thin layers. Hence, following this argument, the development of the instability should be always 


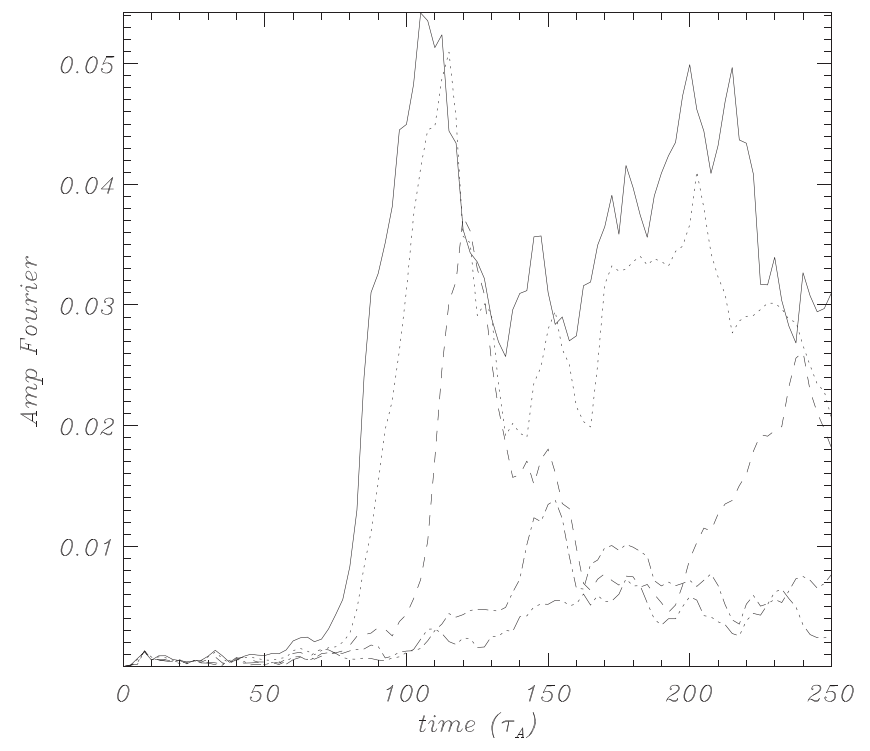

Figure 16. Sum of Fourier coefficients, $\sum_{p=3}^{N / 2}|G(p)|$, as a function of time for the radial velocity $v_{r R}$. The solid, dotted, dashed, dot-dashed, and triple-dotdashed lines correspond to the cases with $B_{\phi} / B_{z}=0,0.1,0.2,0.3$, and 0.4 , respectively. In this case, $l / R=0.3$.

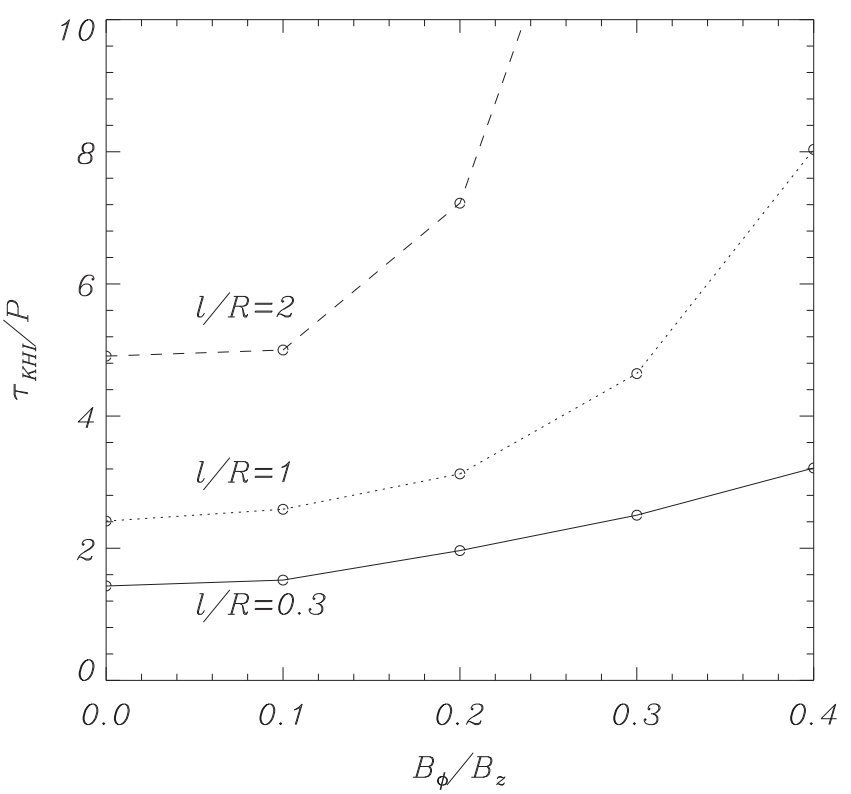

Figure 17. Onset time of the KHI normalized to the kink period as a function of twist calculated from Figure 16 for an inhomogeneous layer of $l / R=0.3$. The results for thicker layers, $l / R=1$ and $l / R=2$, are also shown.

faster when $l / R$ decreases. However, the shear length scales are not the only factor that determines the growth of the instability; the amplitude of the shear is also relevant. This amplitude depends on the energy transference to the inhomogeneous layer, and it grows faster when $l / R$ increases. The two effects are superimposed, but from our results it seems that the generation of small length scales dominates, since we always find that the instability develops faster when $l / R$ decreases. This behavior is enhanced by the effect of twist. From Figure 17 we also find that the dependence of the onset of the instability with twist is stronger for thick layers (compare the curves for $l / R=0.3$ and $l / R=2$ ). Therefore, the combination of significantly wide layers and strong twist produces the most stable configurations regarding the KHI.
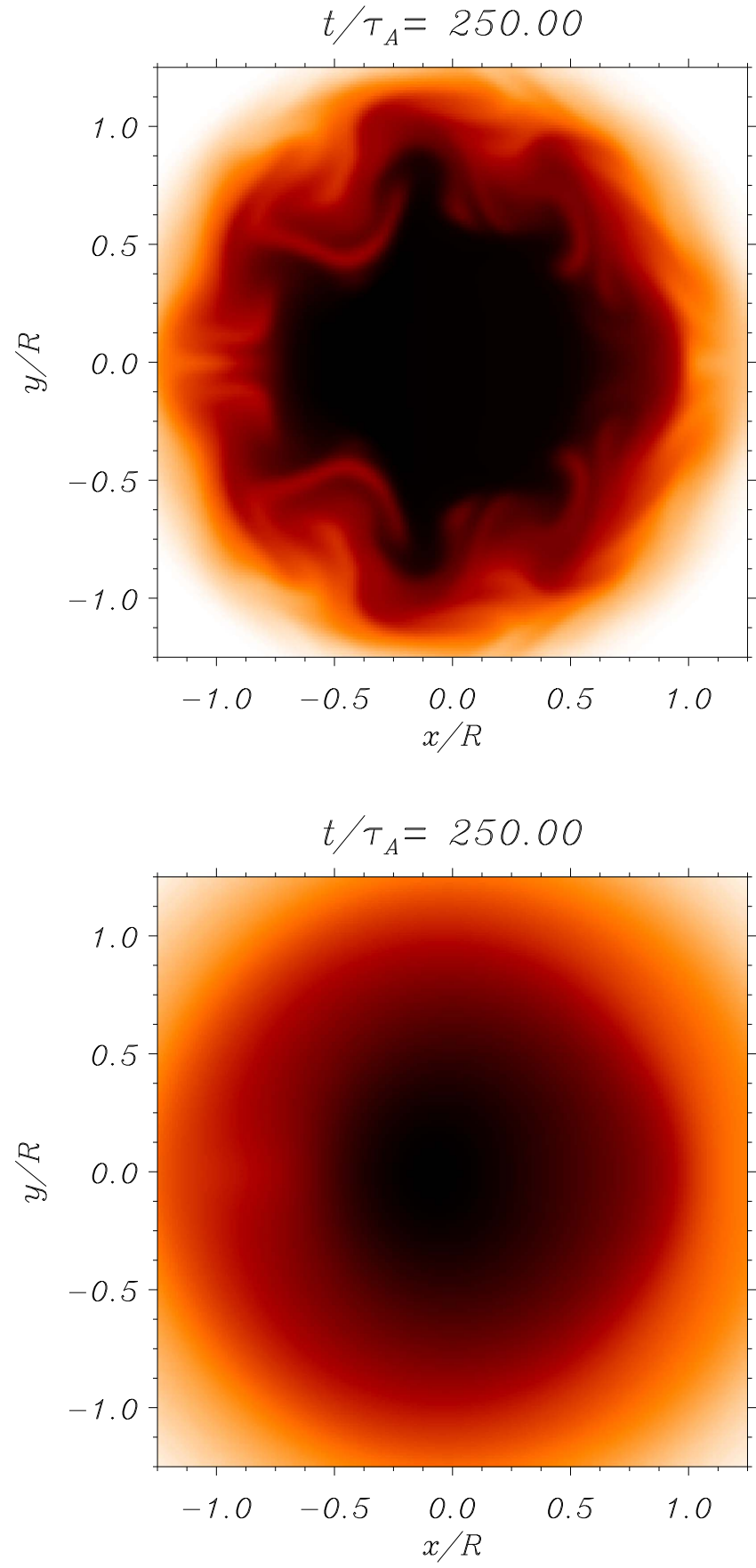

Figure 18. Snapshot of the two-dimensional density distribution at half the tube length $(z=L / 2)$ at a given time. In the top panel $l / R=1$, while in the bottom panel $l / R=2$ (fully inhomogeneous loop). For these simulations, $B_{\phi} / B_{z}=0.2$.

However, we have to bear in mind that tubes with thick layers have much shorter damping times than for the case with thin layers, and the global displacement of the tube is strongly attenuated after a few periods of oscillation. Under such circumstances the instability will develop after the global oscillation has died away. For this reason it is also interesting to compare the timescales of the attenuation, i.e., the damping time, $\tau_{\mathrm{D}}$, with the onset times for the KHI. The results are displayed in Figure 19. From this figure we conclude that only for thin layers the development of the instability can be expected, while the tube is clearly oscillating laterally (i.e., the 


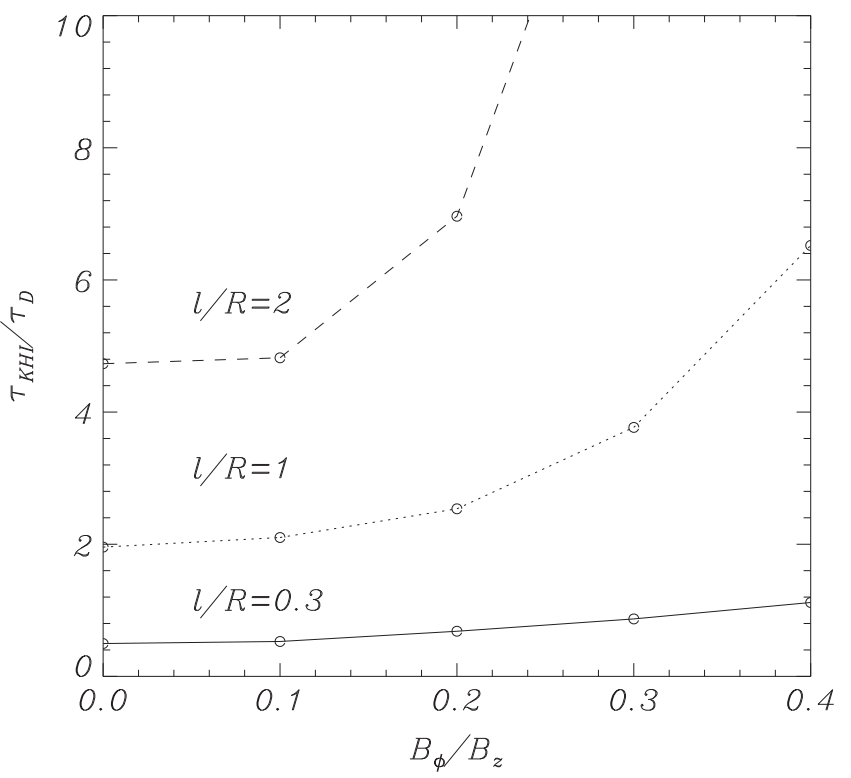

Figure 19. Ratio of KHI onset time to the damping time as a function of twist for three different widths of the inhomogeneous layer. The same parameters as in Figure 17 are used.

situation $\tau_{\mathrm{KHI}} / \tau_{\mathrm{D}}<1$ ), while for the case of thick layers and strong twist the development of the instability takes place after the global transverse oscillation has a negligible amplitude. Note that these results depend on the amplitude of the initial excitation $\left(\xi_{0} / R=0.4\right.$ in the present case), but the chosen value can be viewed as representative of typical transverse loop oscillations, although larger values are usually reported (see Goddard \& Nakariakov 2016, for a detailed study).

Unfortunately, it has not been possible to infer the onset times for the instability using the semianalytic approach used in Section 5.2.1 for the untwisted tube. The reason is that the presence of the magnetic field changes the nature of the Rayleigh equation that has to be solved and the methods used by Heyvaerts \& Priest (1983) and Browning \& Priest (1984) are not easily extended to this situation.

\subsubsection{Effect of Numerical Resolution}

In this section about twist we have used the intermediate resolution, i.e., [400, 400, 80]. However, the nature of the oscillations in this case is not as simple as in the untwisted case, characterized by a dominant large wavelength along the tube. Twist introduces helical motions that produce small wavelengths along the tube, and for this reason it is interesting to check the effect of an increased resolution on the instability onset times. We have repeated the same simulations as in Figure 16 but using [400, 400, 320] points. The results are shown in Figure 20 and indicate that for the values of twist of $B_{\phi} / B_{z}=0,0.1$, and 0.2 the onset times are essentially the same as in the case $[400,400,80]$ (compare with Figure 16). We already found this behavior in the untwisted case (see Section 5.2.2). The differences arise in the situation of strong twist, $B_{\phi} / B_{z}=0.3$ and 0.4 , indicating that insufficient resolution along the tube delays the appearance of the instability. Therefore, increasing the resolution in the $z$-direction slightly reduces the slopes of the curves in Figures 17 and 19. Further evidence of the structuring in the $z$-direction is found in the longitudinal density distribution

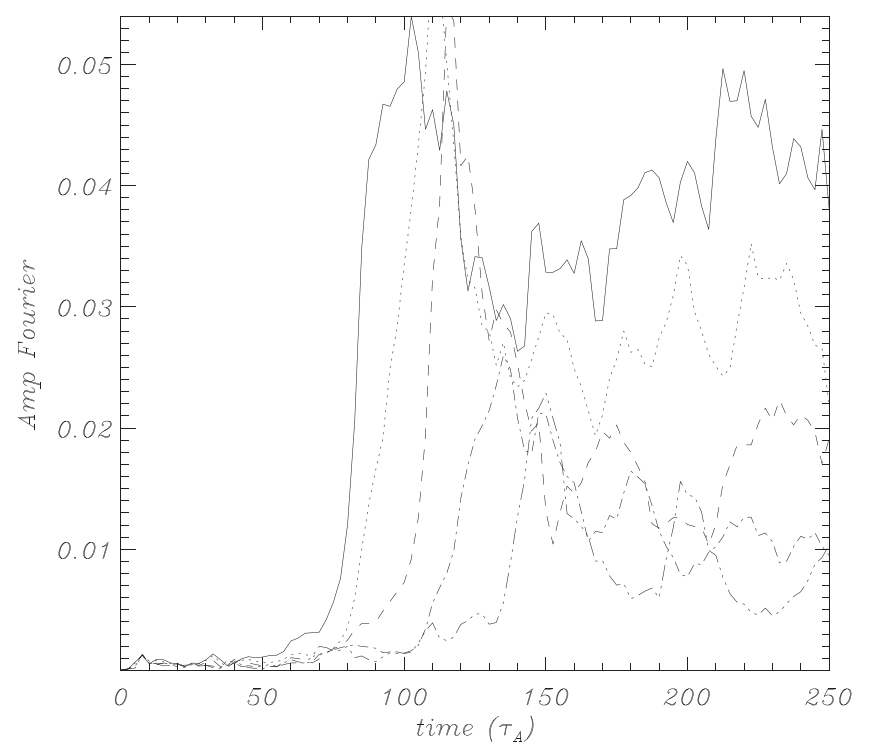

Figure 20. Same as in Figure 16, but in the $z$-direction 320 points, instead of 80 , have been used.

represented in Figure 21. The vortices are present along the loop, contrary to the situation for the untwisted case.

\section{Conclusions and Discussion}

In this work we have investigated numerically the development of the KHI at the edge of transversally $(m=1)$ oscillating tubes. By means of numerical simulations and specific analysis methods to interpret the contribution of the different azimuthal wavenumbers we have extracted several conclusions about the physics involved in this problem. In the numerical experiments, we have found a clear excitation of the azimuthal number $p=2$, with half the period of the transverse oscillation. This result corroborates the analytic predictions of Ruderman et al. (2010) and Ruderman \& Goossens (2014). However, according to our simulations the excitation of the first fluting, $p=2$, does not produce a faster attenuation of the $m=1$ because of the driven nature of this fluting oscillation produced by the nonlinear terms in the MHD equations. The excitation of $p=2$ is essentially unaltered when magnetic twist is included in the model.

Contrary to the initial predictions of Soler et al. (2010) about the stabilizing effect of a weak magnetic twist using a Cartesian slab model (see also the work of Zaqarashvili et al. 2015, in a cylindrical model), we have found that at least for thin inhomogeneous layers a small twist does not significantly delay the appearance of the instability. The same results apply to the case with a discontinuous density profile, which is always the most unstable configuration. Under such conditions the instability might develop during the weakly attenuated global oscillations. This agrees with the results of the submitted paper of Howson et al. (2017), in which further details about the possible enhanced contribution to the heating due to greater ohmic dissipation produced by twist are investigated. However, the observations of damped coronal loop oscillations usually show that the damping is quite strong since the damping per period is typically in the range $1<\tau_{\mathrm{D}} / P<3$ (for example, TRACE oscillations have periods of the order of 2-10 minutes and short damping times of the order of 3-20 minutes; see also the recent study of Goddard \& Nakariakov 2016). In any case 


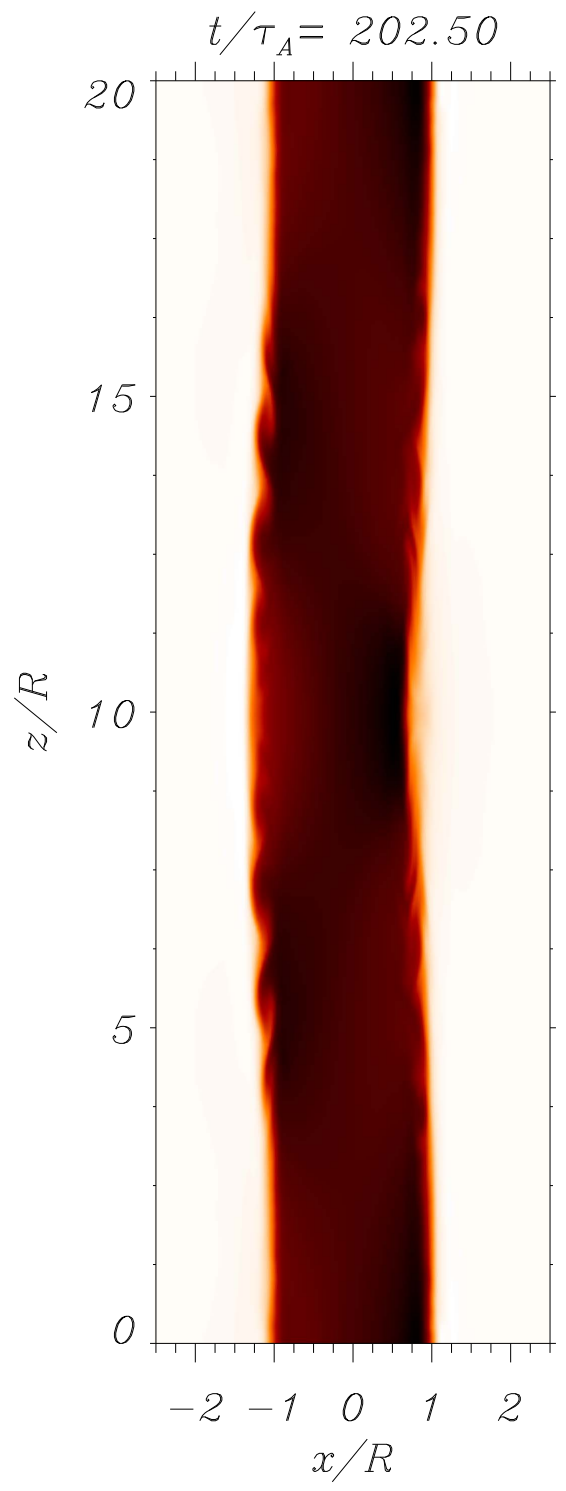

Figure 21. Snapshot of the two-dimensional density distribution (at the plane $y=0)$ at a given time. In this case, $l / R=0.3$ and $B_{\phi} / B_{z}=0.4$.

one needs to be careful when interpreting the damping times from observations, since these times may strongly depend on the amplitude of the perturbation, the EUV channel in which they are detected, the temperature difference between the inside and outside of the loop, and also the resolution of the instrument. For instance, Antolin et al. (2016, 2017) describe apparent decayless oscillations and different damping times depending on the EUV channel and instrument resolution.

Our study indicates that when the inhomogeneous layer that connects the interior of the tube with the external medium is significantly wide (i.e., thick layers), the effect of twist is relevant to the stabilization of the tube with respect to the KHI. The detection of the instability in such a case is expected to be difficult since it is triggered on a timescale much longer than the characteristic damping time of the global oscillation, and the loop is barely oscillating transversally with a significant amplitude. But it may happen that a seemingly static loop suddenly starts developing the KHI if it has been displaced transversally before the beginning of the observations.
Better observations with high spatial resolution are still required to properly resolve the inhomogeneous layers of oscillating magnetic tubes and to provide a definitive answer about the role of resonant damping in the attenuation of the oscillations and therefore in the development of the KHI and the associated heating. In this regard, Okamoto et al. (2015) claim to have found a compelling signature of resonant damping on high spatial, temporal, and spectral resolution observations of a solar prominence (a thread) and point to the relevant role of the $\mathrm{KHI}$ in these observations. Along this line, Kuridze et al. (2015) have recently observed the fast disappearance of rapid redshifted and blueshifted excursions in the $\mathrm{H} \alpha$ line that has been interpreted as a consequence of the heating of the structures due to the development of the KHI at their boundary during transverse motions.

J.T. acknowledges support from MINECO and UIB through a Ramón y Cajal grant and also support by the Spanish MINECO and FEDER funds through project AYA201454485-P. N.M. acknowledges the Fund for Scientific Research-Flanders (FWO-Vlaanderen). T.V.D. was supported by an Odysseus grant of the FWO-Vlaanderen, the IAP P7/08 CHARM (Belspo), and the GOA-2015-014 (KU Leuven). This project has received funding from the European Research Council (ERC) under the European Union's Horizon 2020 research and innovation program (grant agreement no. 724326). The authors also thank R. Soler and M. Goossens for their comments and suggestions that helped to improve the paper. The support from ISSI (International Space Science Institute) through the team "Toward Dynamic Solar Atmospheric Magneto-Seismology with New Generation Instrumentation" led by R. Morton and G. Verth is also acknowledged.

\section{ORCID iDs}

\section{J. Terradas (10) https://orcid.org/0000-0002-7013-1933}

\section{References}

Allan, W., \& Wright, A. N. 1997, JGR, 102, 19927

Antolin, P., De Moortel, I., Van Doorsselaere, T., \& Yokoyama, T. 2017, ApJ, 836,219

Antolin, P., Okamoto, T. J., De Pontieu, B., et al. 2015, ApJ, 809, 72

Antolin, P., Yokoyama, T., \& Van Doorsselaere, T. 2014, ApJL, 787, L22

Antolin, P., Yokoyama, T., \& Van Doorsselaere, T. 2016, ApJL, 830, L22

Aschwanden, M. J. 2013, SoPh, 287, 369

Aschwanden, M. J., Reardon, K., \& Jess, D. B. 2016, ApJ, 826, 61

Aschwanden, M. J., Xu, Y., \& Jing, J. 2014, ApJ, 797, 50

Browning, P. K., \& Priest, E. R. 1984, A\&A, 131, 283

Chandrasekhar, S. (ed.) 1961, Hydrodynamic and Hydromagnetic Stability (Oxford: Clarendon)

Giagkiozis, I., Goossens, M., Verth, G., Fedun, V., \& Van Doorsselaere, T. 2016, ApJ, 823, 71

Goddard, C. R., \& Nakariakov, V. M. 2016, A\&A, 590, L5

Goossens, M., Andries, J., \& Aschwanden, M. J. 2002, A\&A, 394, L39

Goossens, M., Andries, J., Soler, R., et al. 2012, ApJ, 753, 111

Goossens, M., Hollweg, J. V., \& Sakurai, T. 1992, SoPh, 138, 233

Goossens, M., Ruderman, M. S., \& Hollweg, J. V. 1995, SoPh, 157, 75

Heyvaerts, J., \& Priest, E. R. 1983, A\&A, 117, 220

Hollweg, J. V., \& Yang, G. 1988, JGR, 93, 5423

Hood, A. W., \& Priest, E. R. 1979, SoPh, 64, 303

Hood, A. W., Ruderman, M., Pascoe, D. J., et al. 2013, A\&A, 551, A39

Howson, T., De Moortel, I., \& Antolin, P. 2017, A\&A, 607, A77

Karami, K., \& Bahari, K. 2010, SoPh, 263, 87

Kuridze, D., Henriques, V., Mathioudakis, M., et al. 2015, ApJ, 802, 26

Magyar, N., \& Van Doorsselaere, T. 2016, A\&A, 595, A81

Magyar, N., Van Doorsselaere, T., \& Marcu, A. 2015, A\&A, 582, A117

Mann, I. R., Wright, A. N., \& Cally, P. S. 1995, JGR, 100, 19441

Okamoto, T. J., Antolin, P., De Pontieu, B., et al. 2015, ApJ, 809, 71 
Pascoe, D. J., Hood, A. W., de Moortel, I., \& Wright, A. N. 2012, A\&A, 539, A37

Poedts, S., \& Kerner, W. 1991, PhRvL, 66, 2871

Rankin, R., Frycz, P., Tikhonchuk, V. T., \& Samson, J. C. 1994, JGR, 99, 21291

Ruderman, M. S. 2007, SoPh, 246, 119

Ruderman, M. S. 2015, A\&A, 575, A130

Ruderman, M. S. 2017, SoPh, 292, 111

Ruderman, M. S., \& Goossens, M. 2014, SoPh, 289, 1999

Ruderman, M. S., Goossens, M., \& Andries, J. 2010, PhP1, 17, 082108

Ruderman, M. S., \& Roberts, B. 2002, ApJ, 577, 475

Ruderman, M. S., \& Terradas, J. 2013, A\&A, 555, A27

Ruderman, M. S., \& Terradas, J. 2015, A\&A, 580, A57

Sakurai, T., Goossens, M., \& Hollweg, J. V. 1991, SoPh, 133, 227
Soler, R., Goossens, M., Terradas, J., \& Oliver, R. 2013, ApJ, 777, 158

Soler, R., Goossens, M., Terradas, J., \& Oliver, R. 2014, ApJ, 781, 111

Soler, R., Terradas, J., Oliver, R., Ballester, J. L., \& Goossens, M. 2010, ApJ, 712,875

Terradas, J., Andries, J., Goossens, M., et al. 2008, ApJL, 687, L115

Terradas, J., \& Goossens, M. 2012, A\&A, 548, A112

Terradas, J., \& Ofman, L. 2004, ApJ, 610, 523

Terradas, J., Oliver, R., \& Ballester, J. L. 2006, ApJ, 642, 533

Terradas, J., Soler, R., Luna, M., et al. 2016, ApJ, 820, 125

Van Doorsselaere, T., Andries, J., Poedts, S., \& Goossens, M. 2004, ApJ, 606,1223

Wright, A. N., \& Rickard, G. J. 1995, ApJ, 444, 458

Zaqarashvili, T. V., Zhelyazkov, I., \& Ofman, L. 2015, ApJ, 813, 123 